\title{
UNIFORM-IN-TIME SUPERCONVERGENCE OF THE HDG METHODS FOR THE ACOUSTIC WAVE EQUATION
}

\author{
BERNARDO COCKBURN AND VINCENT QUENNEVILLE-BÉLAIR
}

\begin{abstract}
We present the first a priori error analysis of the hybridizable discontinuous Galerkin methods for the acoustic equation in the time-continuous case. We show that the velocity and the gradient converge with the optimal order of $k+1$ in the $L^{2}$-norm uniformly in time whenever polynomials of degree $k \geq 0$ are used. Finally, we show how to take advantage of this local postprocessing to obtain an approximation to the original scalar unknown also converging with order $k+2$ for $k \geq 1$. This puts on firm mathematical ground the numerical results obtained in J. Comput. Phys. 230 (2011), 3695-3718.
\end{abstract}

\section{INTRODUCTION}

In this paper, we present the first theoretical justification of the superconvergence properties of the hybridizable discontinuous Galerkin (HDG) method introduced in [47] for the acoustic wave equation

$$
\rho(x) u_{t t}(t, x)-\nabla \cdot(A \nabla u(x, t))=f(t, x),
$$

with the initial and boundary conditions

$$
\begin{aligned}
u(0, x) & =u^{0}(x), & u_{t}(0, x) & =v^{0}(x), \\
u\left(t, y_{D}\right) & =u_{D}\left(t, y_{D}\right), & \boldsymbol{n} \cdot A \nabla u\left(t, y_{N}\right) & =g_{N}\left(t, y_{N}\right),
\end{aligned}
$$

for $x \in \Omega, t \in[0, T]$, for some $T>0, y_{D} \in \partial \Omega_{D}$, and $y_{N} \in \partial \Omega_{N}$, where $\partial \Omega_{D}$ and $\partial \Omega_{N}$ partition $\partial \Omega$. We take $\Omega$ to be a polyhedral domain. We note that $A=A(x)$ is a symmetric positive definite matrix with $C^{1}(\Omega)$ entries independent of time, and that $\rho(x)>0$ for all $x \in \Omega$. We assume that $\|\rho\|_{W^{1, \infty}(\Omega)},\|A\|_{L^{\infty}(\Omega)}$, and $\|c\|_{L^{\infty}(\Omega)}$ are finite, where $c=A^{-1}$.

The acoustic wave equation is essential in modeling many physical phenomena, thus finding application in many critical engineering fields. Predicting earthquakes and various seismic activity relies heavily on numerical simulations based on solving this equation. Propagating acoustic waves numerically, for either noise reduction or radar detection, generates significant interest.

This paper is part of the development of the HDG methods which were introduced in the framework of diffusion problems [15] as an alternative to the already

Received by the editor October 14, 2011 and, in revised form, January 26, 2012.

2010 Mathematics Subject Classification. Primary 65M60, 65N30, 65M15, 65M22.

Key words and phrases. Discontinuous Galerkin methods, hybridization, superconvergence, hyperbolic problems.

The first author was partially supported by the National Science Foundation (Grant DMS1115331) and by the Minnesota Supercomputing Institute. 
existing DG methods. The new HDG methods display a significantly smaller number of globally coupled unknowns and are more accurate, as was proven later in [13, 17, 18, 21]. The methods were then extended to the convection-diffusion equations [10, 50, 51, to the heat equation [9], to linear and nonlinear elasticity [57, 58, to the biharmonic [14, to the Timoshenko beam model [7,8], to the Stokes equations [12, 16, 20, 22, 23, 52, and to the incompressible [46, 48, and compressible [54] Navier-Stokes equations; see also the review [49].

The method was also extended to wave propagation in acoustics and elastodynamics in [47. As the numerical experiments therein illustrate, the velocity and the gradient converge with the optimal order of $k+1$ in the $L^{2}$-norm whenever polynomials of degree $k \geq 0$ are used. Moreover, an inexpensive local postprocessing enables superconvergence in the $L^{2}$-norm of order $k+2$ for the displacement with polynomials of degree $k \geq 1$. In this paper, we carry out the first theoretical analysis of the HDG methods for acoustics.

Let us place our results in perspective. Thus far, time-dependent acoustic wave propagation has been tackled using various techniques, including finite difference methods, integral methods, and finite element methods. Finite difference methods are popular $[1,25,29,42,44,62,63$ due to their simplicity of implementation. However, they have difficulties handling non-regular grids or complex geometries. This makes it difficult to do adaptivity in contrast to integral equation and finite element methods.

The integral equation methods rely on writing the equation of interest in an integral form. In fact, the wave equation on a piecewise homogeneous domain can be written in an integral form involving only the boundary of the domain and subdomains. This reduction in dimensionality is the main advantage of integral equation methods, as explained in the review by Nishimura in 2002 [53. Indeed, this substantially decreases the number of unknowns. Unfortunately, the resulting matrix is dense and might be so inefficient to invert numerically that the benefit of the dimensionality reduction might be lost. To address this, many techniques, such as the Fast Multiple Method introduced by Greengard and Rokhlin in 1987 38, have been developed to accelerate the solving of the linear system. The review by Nishimura 53 explains the ideas behind the Fast Multipole Method. When applied to the frequency domain, one can achieve exponential convergence, as detailed in the book by Colton and Kress in 1998 [26]. However, in order to rewrite the equations as boundary integrals, the fundamental solution for the wave equation is needed. Their presence usually leads to singular integrals, so that they need to be evaluated carefully, as detailed by Bruno [5]. Moreover, if the boundary is not smooth, special techniques then have to be employed to handle the integrals, as shown in [6].

In the presence of inhomogeneous media or materials, integral methods cannot be used, and finite element methods, in at least part of the computational domain, can come into play. The finite element methods have the advantage of handling complex geometries and unstructured meshes. This later advantage is especially desirable for adaptivity, which helps focus the computational effort near numerically difficult parts of the domain. Examples are the continuous Galerkin (CG) method [2,30, but also multiple mixed finite element methods [4, 24, 27, 28, 37, 40, 41, and many DG methods [33, 34, 39, 43, 45, 55, as we describe next. 
Dupont [30] and Baker [2] studied CG methods for time-dependent second-order linear hyperbolic problems via energy arguments, and they both showed that the displacement converges with order $k+1$ in the $L^{2}$-norm.

In the case of mixed methods, the acoustic wave equation is recast as a firstorder system for the velocity and the gradient. While increasing the flexibility of the choice of approximations, this allows the computation of some extra physical quantities at the cost of having more unknowns to solve for. Geveci [37] used the Raviart-Thomas and the Brezzi-Douglas-Marini spaces on a formulation similar to the one used here, and proved the convergence of order $k$ in the $L^{2}$-norm for both the velocity and the gradient. Cowsar, Dupont, and Wheeler [27, 28], on the other hand, wrote their formulation for the displacement and the gradient keeping both time derivatives on the displacement. They proved convergence of order $k+1$ in the $L^{2}$-norm for both the velocity and the gradient. Later, and using a different mixed formulation in which the second derivative in time of the vector quantity is the gradient of the scalar, and the scalar is the divergence of the vector, Jenkins, Rivière, and Wheeler 41 obtained estimates of the order $k$ in the vector and the scalar quantity using Raviart-Thomas-Nédélec, Brezzi-Douglas-Marini and the Brezzi-Douglas-Fortin-Marini spaces. Later, Jenkins [40] numerically showed order of convergence of $k+1$ in the $L^{2}$-norm for both the scalar and the vector quantity using the Raviart-Thomas element on rectangles (and for $k=0,1$ ) with this formulation.

In the case of the DG methods, the system is often written as a symmetric firstorder hyperbolic system [33, 34, 45] where the unknown is a vector. Indeed, in such a form, Falk and Richter 34 and then Monk and Richter 45 obtained convergence of order $k+1 / 2$ for the $L^{2}$-norm of the vector. Using the symmetric interior penalty method, Gröte, Schneebeli, and Schötzau [39] obtained convergence in the $L^{2}$-norm of order $k+1$ for the displacement when applying the method directly to the secondorder wave equation. Table 1.1 shows a comparison of some finite element methods mentioned above.

This paper carries out the analysis of the method for the acoustic equation in the time continuous case for the HDG method introduced in 47. Indeed, we show that we obtain optimal convergence of order $k+1$ in the $L^{2}$-norm for the velocity and the gradient - that is, the method is competitive with the other finite element methods. Moreover, a simple inexpensive local postprocessing improves the result for the displacement, and a superior convergence in the $L^{2}$-norm of order $k+2$ is achieved uniformly in time, provided that the entries of $A$ are $C^{1}(\Omega)$ and that the initial conditions are imposed using a specific projection. The particular HDG method considered is the local discontinuous Galerkin-hybridizable (LDG-H) method, as detailed in 15.

The proof of the convergence properties relies on a new projection-based technique introduced in [17] for diffusion problems. This technique enables us to analyze the projection of the error through an energy argument, and, more importantly, the superconvergence through a duality argument. Indeed, duality arguments in combination with a projection-based approach have been successfully employed for the diffusion [13, 17, convection-diffusion, and heat 9] equations, to the Stokes problem [16], to the Timoshenko beam model [7, and to the biharmonic equation [14]. In sharp contrast with all the above-mentioned problems, ours is a purely hyperbolic 
TABLE 1.1. Comparison of local spaces and proven orders of convergence in $L^{2}$ of finite element methods for smooth solutions of the acoustic wave equation. The superscript ${ }^{\star}$ denotes a postprocessing.

\begin{tabular}{|c|c|c|c|c|c|c|}
\hline \multirow{2}{*}{ Method } & \multicolumn{3}{|c|}{ Local Spaces } & \multicolumn{3}{|c|}{ Order } \\
\hline & $u_{h}$ & $v_{h}$ & $\boldsymbol{q}_{h}$ & $u_{h}$ & $v_{h}$ & $\boldsymbol{q}_{h}$ \\
\hline$\underset{k \geq 1}{\mathrm{CG}[2,30]}$ & $\mathcal{P}_{k}$ & - & - & $k+1$ & - & - \\
\hline $\begin{array}{c}\mathrm{IP}[39] \\
k \geq 1\end{array}$ & $\mathcal{P}_{k}$ & - & - & $k+1$ & - & - \\
\hline$\underset{k \geq 0}{\operatorname{Mixed}}[27,28]$ & $\mathcal{P}_{k}$ & - & $\mathrm{RT}_{k}$ & $k+1$ & - & $k+1$ \\
\hline Mixed 37. & - & $\begin{array}{c}\mathcal{P}_{k} \\
\mathcal{P}_{k-1}\end{array}$ & $\begin{array}{c}\mathrm{RT}_{k} \\
\mathrm{BDM}_{k}\end{array}$ & - & $\begin{array}{c}k+1 \\
k\end{array}$ & $\begin{array}{c}k+1 \\
k\end{array}$ \\
\hline DG $\underset{k \geq 0}{34}, 45$ & - & $\mathcal{P}_{k}$ & $\left(\mathcal{P}_{k}\right)^{d}$ & - & $k+1 / 2$ & $k+1 / 2$ \\
\hline$\underset{k \geq 0}{\mathrm{HDG}}$ & - & $\mathcal{P}_{k}$ & $\left(\mathcal{P}_{k}\right)^{d}$ & - & $k+1$ & $k+1$ \\
\hline \multirow[t]{2}{*}{$\underset{k \geq 1}{\mathrm{HDG}}$} & $\mathcal{P}_{k+1}$ & - & $\mathrm{RT}_{k}$ & $k+2$ & - & $k+1$ \\
\hline & $u_{h}^{\star}$ & - & $\boldsymbol{q}_{h}^{\star}$ & $u_{h}^{\star}$ & - & $\nabla \cdot \boldsymbol{q}_{h}^{\star}$ \\
\hline
\end{tabular}

problem and lacks the regularization mechanism typical of elliptic equations. Nevertheless, we show that we can still make strong use of elliptic regularity results to prove the superconvergence properties of the method.

The paper is organized as follows. In Section 2, we introduce the HDG method, the projection used in the analysis, and we present and discuss the estimates for the error of the approximation. In Section 3, we display all the proofs. We end in Section 4 with some extensions and concluding remarks.

\section{MAIN RESUlts}

2.1. The HDG approximation to $u_{t}$ and $A \nabla u$. To define the HDG method, we follow [47]. Thus, we introduce the auxiliary variables $v:=u_{t}$ and $\boldsymbol{q}:=A \nabla u$ and rewrite the wave equation as a first-order system

$$
\begin{aligned}
\rho v_{t}-\nabla \cdot \boldsymbol{q} & =f, \\
c \boldsymbol{q}_{t}-\nabla v & =0,
\end{aligned}
$$

and complete it with the initial and boundary conditions

$$
\begin{aligned}
v(0, x) & =v^{0}(x), & \boldsymbol{q}(0, x)=A \nabla u^{0}(x) & =: \boldsymbol{q}^{0}(x), \\
v\left(t, y_{D}\right) & =\left(u_{D}\right)_{t}\left(t, y_{D}\right)=: g_{D}\left(t, y_{D}\right), & \boldsymbol{n} \cdot \boldsymbol{q}\left(t, y_{N}\right) & =g_{N}\left(t, y_{N}\right),
\end{aligned}
$$

for $x \in \Omega, t \in[0, T], y_{D} \in \partial \Omega_{D}$, and $y_{N} \in \partial \Omega_{N}$.

We discretize the above problem in space as follows. First, we discretize our domain $\Omega$ by a conforming triangulation $\mathcal{T}_{h}$ made of simplexes. We define $\mathcal{E}_{h}$ to be the set of all faces $F$ of the simplexes $K$ of the triangulation $\mathcal{T}_{h}$. We assume that, if a face $F$ is on $\partial \Omega$, then either $F$ is completely inside $\partial \Omega_{D}$ or completely inside $\partial \Omega_{N}$. For each element $K$ of the triangulation $\mathcal{T}_{h}$, we define $(f, g)_{K}:=\int_{K} f g$, 
$(\boldsymbol{f}, \boldsymbol{g})_{\mathcal{T}_{h}}:=\sum_{K \in \mathcal{T}_{h}} \int_{K} \boldsymbol{f} \cdot \boldsymbol{g}$, and then set $\langle f, g\rangle_{\partial \mathcal{T}_{h}}:=\sum_{K \in \mathcal{T}_{h}} \int_{\partial K} f g$. We denote by $\boldsymbol{n}$ the unit outward normal to $K$.

For each time $t \in[0, T]$, the HDG method provides an approximation to $\left(\left.\boldsymbol{q}(t)\right|_{\Omega},\left.v(t)\right|_{\Omega},\left.v(t)\right|_{\mathcal{E}_{h}}\right)$, which we denote by $\left(\left.\boldsymbol{q}_{h}(t)\right|_{\Omega},\left.v_{h}(t)\right|_{\Omega},\left.\widehat{v}_{h}(t)\right|_{\mathcal{E}_{h}}\right)$, lying in the space $\boldsymbol{V}_{h} \times W_{h} \times M_{h}$, where

$$
\begin{aligned}
& W_{h}=\left\{w \in L^{2}(\Omega):\left.\quad w\right|_{K} \in \mathcal{P}_{k}(K) \quad \forall K \in \mathcal{T}_{h}\right\}, \\
& \boldsymbol{V}_{h}=\left\{\boldsymbol{q} \in\left(L^{2}(\Omega)\right)^{d}:\left.\boldsymbol{q}\right|_{K} \in\left(\mathcal{P}_{k}(K)\right)^{d} \forall K \in \mathcal{T}_{h}\right\}, \\
& M_{h}=\left\{\mu \in L^{2}\left(\mathcal{E}_{h}\right):\left.\quad \mu\right|_{F} \in \mathcal{P}_{k}(F) \quad \forall F \in \mathcal{E}_{h}\right\} .
\end{aligned}
$$

The approximation is determined as the solution of

$$
\begin{aligned}
\left(c\left(\boldsymbol{q}_{h}\right)_{t}, \boldsymbol{r}\right)_{\mathcal{T}_{h}}+\left(v_{h}, \nabla \cdot \boldsymbol{r}\right)_{\mathcal{T}_{h}}-\left\langle\widehat{v}_{h}, \boldsymbol{r} \cdot \boldsymbol{n}\right\rangle_{\partial \mathcal{T}_{h}} & =0, \\
\left(\rho\left(v_{h}\right)_{t}, w\right)_{\mathcal{T}_{h}}+\left(\boldsymbol{q}_{h}, \nabla w\right)_{\mathcal{T}_{h}}-\left\langle\widehat{\boldsymbol{q}}_{h} \cdot \boldsymbol{n}, w\right\rangle_{\partial \mathcal{T}_{h}} & =(f, w)_{\mathcal{T}_{h}}, \\
\left\langle\widehat{\boldsymbol{q}}_{h} \cdot \boldsymbol{n}, \mu\right\rangle_{\partial \mathcal{T}_{h} \backslash \partial \Omega} & =0,
\end{aligned}
$$

for all $(\boldsymbol{r}, w, \mu) \in \boldsymbol{V}_{h} \times W_{h} \times M_{h}$. The numerical trace $\widehat{\boldsymbol{q}}_{h}$ is given by

$$
\widehat{\boldsymbol{q}}_{h}=\boldsymbol{q}_{h}-\tau\left(v_{h}-\widehat{v}_{h}\right) \boldsymbol{n} \quad \text { on } \partial \mathcal{T}_{h},
$$

where the stabilization function $\tau: \partial \mathcal{T}_{h} \mapsto \mathbb{R}^{+}$is constant on each face of each element $K \in \mathcal{T}_{h}$.

The initial conditions are discretized as

$$
\begin{aligned}
& \boldsymbol{q}_{h}(x, 0)=\boldsymbol{P}_{\boldsymbol{V}} \boldsymbol{q}^{0}(x), \\
& v_{h}(x, 0)=P_{W} v^{0}(x),
\end{aligned}
$$

where $\boldsymbol{P}_{\boldsymbol{V}}$ and $P_{W}$ are suitably chosen projections into $\boldsymbol{V}_{h}$ and $W_{h}$, respectively. Finally the boundary conditions are imposed weakly as

$$
\begin{aligned}
\left\langle\widehat{v}_{h}, \mu\right\rangle_{\partial \Omega_{D}} & =\left\langle g_{D}, \mu\right\rangle_{\partial \Omega_{D}} \\
\left\langle\widehat{\boldsymbol{q}}_{h} \cdot \boldsymbol{n}, \mu\right\rangle_{\partial \Omega_{N}} & =\left\langle g_{N}, \mu\right\rangle_{\partial \Omega_{N}},
\end{aligned}
$$

for all $\mu \in M_{h}$. This completes the definition of the HDG methods.

2.2. New approximations. Next, we define postprocessings of the approximate solution provided by the HDG method, which provides new approximations to the flux and the original scalar unknown $u$. The postprocessing of the flux provides a new approximation $\boldsymbol{q}_{h}^{\star}$ which, unlike $\boldsymbol{q}_{h}$, lies in the space $\boldsymbol{H}(\operatorname{div}, \Omega)$ and converges with the same order as $\boldsymbol{q}_{h}$; it is defined by using a slight modification of the RaviartThomas projection [56] (see also [3, 19] and [17,18]). Finally, the approximation for $u, u_{h}^{\star}$, which converges with one additional order than $u_{h}$ for $k \geq 1$, is obtained by evolving in time the local averages of $u$ and by using them in the postprocessing introduced in $36,59,60$.

Postprocessing of the flux, $\boldsymbol{q}_{h}^{\star}$. Thus, on each simplex $K \in \mathcal{T}_{h}$, we take $\boldsymbol{q}_{h}^{\star}$ to be the only element of $\left(\mathcal{P}_{k}(K)\right)^{d}+\boldsymbol{x} \mathcal{P}_{k}(K)$ satisfying

$$
\begin{aligned}
& \left(\boldsymbol{q}_{h}^{\star}, \boldsymbol{v}\right)_{K}=\left(\boldsymbol{q}_{h}, \boldsymbol{v}\right)_{K} \quad \forall \boldsymbol{v} \in\left(\mathcal{P}_{k-1}(K)\right)^{d}, \\
& \left\langle\boldsymbol{q}_{h}^{\star} \cdot \boldsymbol{n}, \mu\right\rangle_{F}=\left\langle\widehat{\boldsymbol{q}}_{h} \cdot \boldsymbol{n}, \mu\right\rangle_{F} \quad \forall \mu \in \mathcal{P}_{k}(F) \text { and all faces } F \text { of } K \text {. }
\end{aligned}
$$


The approximation $u_{h}^{\star}$. We compute an approximation to $u, u_{h}^{\star}$, as follows. For each element $K \in \mathcal{T}_{h}$, we evolve in time the average of $u_{h}$ on $K$ by solving

$$
\begin{aligned}
\frac{d}{d t}\left(u_{h}, 1\right)_{K} & =\left(v_{h}, 1\right)_{K}, \\
\left(u_{h}(0), 1\right)_{K} & =\left(u_{0}, 1\right)_{K} .
\end{aligned}
$$

We then compute $u_{h}^{\star}$ on $K$ as the only function in $\mathcal{P}_{k+1}(K)$ satisfying

$$
\begin{aligned}
\left(\nabla u_{h}^{\star}, \nabla w\right)_{K} & =\left(c \boldsymbol{q}_{h}, \nabla w\right)_{K} \quad \forall w \in \mathcal{P}_{k+1}(K), \\
\left(u_{h}^{\star}, 1\right)_{K} & =\left(u_{h}, 1\right)_{K} .
\end{aligned}
$$

\subsection{The a priori error estimates.}

The projection of the errors. We provide estimates of the following projection of the errors:

$$
\begin{aligned}
\varepsilon^{q}:=\boldsymbol{\Pi}_{\boldsymbol{V}} \boldsymbol{q}-\boldsymbol{q}_{h} \in \boldsymbol{V}_{h}, & \varepsilon^{v}:=\Pi_{W} v-v_{h} \in W_{h}, \\
\boldsymbol{\varepsilon}^{\widehat{q}} \cdot \boldsymbol{n}:=P_{M}(\boldsymbol{q} \cdot \boldsymbol{n})-\widehat{\boldsymbol{q}}_{h} \cdot \boldsymbol{n} \in M_{h}, & \varepsilon^{\widehat{v}}:=P_{M} v-\widehat{v}_{h} \in M_{h},
\end{aligned}
$$

where $P_{M}$ is the $L^{2}$ projection into $M_{h}$ and where $\left(\boldsymbol{\Pi}_{\boldsymbol{V}}, \Pi_{W}\right)$ is the projection introduced in [17] in the framework of the error analysis of HDG methods for diffusion equations. We recall that the projection $\Pi(\boldsymbol{q}, v)=\left(\boldsymbol{\Pi}_{\boldsymbol{V}} \boldsymbol{q}, \Pi_{W} v\right) \in \boldsymbol{V}_{h} \times$ $W_{h}$ is defined as follows. On any simplex $K$, we require that $\Pi(\boldsymbol{q}, v)$ be the solution of the equations

$$
\begin{aligned}
\left(\boldsymbol{\Pi}_{\boldsymbol{V}} \boldsymbol{q}-\boldsymbol{q}, \boldsymbol{r}\right)_{K} & =0, \\
\left(\Pi_{W} v-v, w\right)_{K} & =0, \\
\left\langle\left(\widehat{\boldsymbol{\Pi}_{\boldsymbol{V}}} \boldsymbol{q}-\boldsymbol{q}\right) \cdot \boldsymbol{n}, \mu\right\rangle_{F} & =0,
\end{aligned}
$$

for all $\boldsymbol{r} \in \mathcal{P}_{k-1}^{d}(K), w \in \mathcal{P}_{k-1}(K)$, and $\mu \in \mathcal{P}_{k}(F)$ for all faces $F$ of $K$, where we set

$$
\widehat{\Pi_{\boldsymbol{V}} \boldsymbol{q}} \cdot \boldsymbol{n}:=\boldsymbol{\Pi}_{\boldsymbol{V}} \boldsymbol{q} \cdot \boldsymbol{n}-\tau\left(\Pi_{W} v-P_{M} v\right) \quad \text { on } \partial K .
$$

Let us also recall the main result about this projection.

Theorem $2.1([17])$. Suppose $k \geq 0,\left.\tau\right|_{\partial K}$ is non-negative and $\tau_{K}^{\max }:=\left.\max \tau\right|_{\partial K}>$ 0 . Then $\left(\boldsymbol{\Pi}_{\boldsymbol{V}} \boldsymbol{q}, \Pi_{W} v\right)$ is well defined. Furthermore, there is a constant $C_{\Pi}$ independent of $K$ and $\tau$ such that

$$
\begin{gathered}
\left\|\boldsymbol{\Pi}_{\boldsymbol{V}} \boldsymbol{q}-\boldsymbol{q}\right\|_{L^{2}(K)} \leq C h_{K}^{l_{q}+1}|\boldsymbol{q}|_{H^{l_{q}+1}(K)}+C h_{K}^{l_{v}+1} \tau_{K}^{\star}|v|_{H^{l_{v}+1}(K)}, \\
\left\|\Pi_{W} v-v\right\|_{L^{2}(K)} \leq C h_{K}^{l_{v}+1}|v|_{H^{l_{v}+1}(K)}+C \frac{h_{K}^{l_{q}+1}}{\tau_{K}^{\max }}|\nabla \cdot \boldsymbol{q}|_{H^{l_{q}(K)}},
\end{gathered}
$$

for $l_{v}, l_{q}$ in $[0, k]$. Here $\tau_{K}^{\star}:=\left.\max \tau\right|_{\partial K \backslash F^{\star}}$, where $F^{\star}$ is a face of $K$ at which $\left.\tau\right|_{\partial K}$ is maximum.

Thus, if $\tau_{K}^{\star}$ and $1 / \tau_{K}^{\max }$ are uniformly bounded for all $K \in \mathcal{T}_{h}$, then the projection converges with order $k+1$ for both variables provided the solution is smooth enough. 
Estimates of the projection of the errors. In order to get our superconvergence result, we need to assume that the domain $\Omega$ is such that the elliptic regularity estimate,

$$
\|\varsigma\|_{H^{2}(\Omega)} \leq C_{\mathrm{reg}}\|\nabla \cdot(A \nabla \varsigma)\|_{L^{2}(\Omega)},
$$

holds for any $\nabla \varsigma \in H_{0}$ (div), the space of functions with divergence in $L^{2}(\Omega)$ and having zero normal component on the border, or any $\varsigma \in H_{0}^{1}(\Omega)$ such that the right-hand side of equation (2.9) is finite. This inequality holds for any convex domain, but not for mixed Dirichlet-Neumann boundary conditions; see, for example, Subsection 3.1.3 [31] and the references therein.

We use the standard notation for the norms and seminorms of Banach and, in particular, Sobolev spaces. Moreover, for $p=1$ and $p=2$, we set, for any Banach space $B,\|f\|_{L^{p}(T ; B)}:=\left(\int_{0}^{T}\|f(t)\|_{B}^{p} d t\right)^{1 / p}$ and $\|f\|_{L^{\infty}(T ; B)}:=\sup _{t \in(0, T)}\|f(t)\|_{B}$. Furthermore, we set $\overline{\varepsilon^{q}}(t)=\int_{0}^{t} \varepsilon^{q}$, and similarly for $\overline{\varepsilon^{v}}, \overline{\varepsilon^{\widehat{v}}}$, and $\overline{\varepsilon^{\widehat{q}}}$.

Finally, we denote by $\mathrm{P}_{\ell}$ the projection that, on each element $K \in \mathcal{T}_{h}$, is nothing but the $L^{2}(K)$-projection into $\mathcal{P}_{\ell}(K)$. Our main result is the following.

Theorem 2.2. For any $T \geq 0$ and $k \geq 0$, we have

$$
\begin{aligned}
& {\left[\left\|\sqrt{c} \varepsilon^{q}\right\|_{L^{\infty}\left(T ; L^{2}\left(\mathcal{T}_{h}\right)\right)}^{2}+\left\|\sqrt{\rho} \varepsilon^{v}\right\|_{L^{\infty}\left(T ; L^{2}\left(\mathcal{T}_{h}\right)\right)}^{2}+2\left\|\sqrt{\tau}\left(\varepsilon^{v}-\varepsilon^{\widehat{v}}\right)\right\|_{L^{2}\left(T ; L^{2}\left(\partial \mathcal{T}_{h}\right)\right)}^{2}\right]^{1 / 2}} \\
& \quad \leq\left[\left\|\Pi_{W} v^{0}-P_{W} v^{0}\right\|_{L^{2}\left(\mathcal{T}_{h}\right)}^{2}+\left\|\boldsymbol{\Pi}_{\boldsymbol{V}} \boldsymbol{q}^{0}-\boldsymbol{P}_{\boldsymbol{V}} \boldsymbol{q}^{0}\right\|_{L^{2}\left(\mathcal{T}_{h}\right)}^{2}\right]^{1 / 2} \\
& \quad+\left\|\sqrt{c}\left(\boldsymbol{\Pi}_{\boldsymbol{V}} \boldsymbol{q}_{t}-\boldsymbol{q}_{t}\right)\right\|_{L^{1}\left(T ; L^{2}\left(\mathcal{T}_{h}\right)\right)}+\left\|\sqrt{\rho}\left(\Pi_{W} v_{t}-v_{t}\right)\right\|_{L^{1}\left(T ; L^{2}\left(\mathcal{T}_{h}\right)\right)} .
\end{aligned}
$$

Moreover, if we take $\left(\boldsymbol{P}_{\boldsymbol{V}}, P_{W}\right):=\left(\boldsymbol{\Pi}_{\boldsymbol{V}}, \Pi_{W}\right)$ as the projection with which we define the initial condition, we have

$$
\begin{aligned}
& {\left[\left\|\sqrt{c} \varepsilon_{t}^{q}\right\|_{L^{\infty}\left(T ; L^{2}\left(\mathcal{T}_{h}\right)\right)}^{2}+\left\|\sqrt{\rho} \varepsilon_{t}^{v}\right\|_{L^{\infty}\left(T ; L^{2}\left(\mathcal{T}_{h}\right)\right)}^{2}+2\left\|\sqrt{\tau}\left(\varepsilon_{t}^{v}-\varepsilon_{t}^{\widehat{v}}\right)\right\|_{L^{2}\left(T ; L^{2}\left(\partial \mathcal{T}_{h}\right)\right)}^{2}\right]^{1 / 2}} \\
& \leq\left[\left\|\Pi_{W} v_{t}(0)-v_{t}(0)\right\|_{L^{2}\left(\mathcal{T}_{h}\right)}^{2}+\left\|\boldsymbol{\Pi}_{\boldsymbol{V}} \boldsymbol{q}_{t}(0)-\boldsymbol{q}_{t}(0)\right\|_{L^{2}\left(\mathcal{T}_{h}\right)}^{2}\right]^{1 / 2} \\
& \quad+\left\|\sqrt{c}\left(\boldsymbol{\Pi}_{\boldsymbol{V}} \boldsymbol{q}_{t t}-\boldsymbol{q}_{t t}\right)\right\|_{L^{1}\left(T ; L^{2}\left(\mathcal{T}_{h}\right)\right)}+\left\|\sqrt{\rho}\left(\Pi_{W} v_{t t}-v_{t t}\right)\right\|_{L^{1}\left(T ; L^{2}\left(\mathcal{T}_{h}\right)\right)} .
\end{aligned}
$$

If, in addition, we have an integer $l$ such that $1 \leq l \leq k$, and the elliptic regularity condition (2.9) holds, we have

$$
\begin{aligned}
\left\|\mathrm{P}_{l-1} \overline{\varepsilon^{v}}(T)\right\|_{L^{2}\left(\mathcal{T}_{h}\right)} \leq C T h( & \left\|\sqrt{\rho}\left(v-v_{h}\right)_{t}\right\|_{L^{\infty}\left(T ; L^{2}\left(\mathcal{T}_{h}\right)\right)} \\
& \left.+\left\|\sqrt{c}\left(\boldsymbol{q}-\boldsymbol{q}_{h}\right)_{t}\right\|_{L^{\infty}\left(T ; L^{2}\left(\mathcal{T}_{h}\right)\right)}\right) .
\end{aligned}
$$

We also have estimates of projections of the errors of the new approximations. In the following result, for each simplex $K \in \mathcal{T}_{h}, \Pi_{k}^{\mathrm{RT}}$ denotes the Raviart-Thomas projection into $\left(\mathcal{P}_{k}(K)\right)^{d}+x \mathcal{P}_{k}(K)$, and $\mathrm{P}_{k+1}$ the $L^{2}$-projection into $\mathcal{P}_{k+1}(K)$.

Proposition 2.1. For any element $K \in \mathcal{T}_{h}$, we have that

$$
\begin{aligned}
\left\|\Pi_{k}^{\mathrm{RT}} \boldsymbol{q}-\boldsymbol{q}_{h}^{\star}\right\|_{L^{2}(K)} \leq & C\left(\left\|\varepsilon^{q}\right\|_{L^{2}(K)}+\left(h_{K}\|\tau\|_{L^{\infty}(\partial K)}\right)^{1 / 2}\left\|\sqrt{\tau}\left(\varepsilon^{v}-\varepsilon^{\widehat{v}}\right)\right\|_{\partial K}\right), \\
\left\|\nabla \cdot\left(\Pi_{k}^{\mathrm{RT}} \boldsymbol{q}-\boldsymbol{q}_{h}^{\star}\right)\right\|_{L^{2}(K)} \leq & C\left\|\left(v-v_{h}\right)_{t}\right\|_{L^{2}(K)}, \\
\left\|\mathrm{P}_{k+1} u-u_{h}^{\star}\right\|_{L^{2}(K)} \leq & C h\left(\left\|\nabla\left(u-\mathrm{P}_{k+1} u\right)\right\|_{L^{2}(K)}+\left\|c\left(\boldsymbol{q}_{h}-\boldsymbol{q}\right)\right\|_{L^{2}(K)}\right) \\
& +\left\|\mathrm{P}_{0} \overline{\varepsilon^{v}}\right\|_{L^{2}(K)} .
\end{aligned}
$$


Estimates of the errors. Using the approximation results of the projection $\left(\boldsymbol{P}_{\boldsymbol{V}}, P_{W}\right)$, Theorem 2.1 and the approximation properties of the projections $\Pi_{k}^{\mathrm{RT}}$ and $\mathrm{P}_{k+1}$ in the estimates of Theorem 2.2 and Proposition 2.1, we get the following result.

Corollary 2.1. Assume that the exact solution $(\boldsymbol{q}, v, u)$ is very smooth. Assume also that $\tau_{K}^{\star}$ and $1 / \tau_{K}^{\max }$ are uniformly bounded for all $K \in \mathcal{T}_{h}$. Then, for any $T \geq 0$ and $k \geq 0$, we have

$$
\left\|v-v_{h}\right\|_{L^{\infty}\left(T ; L^{2}\left(\mathcal{T}_{h}\right)\right)}+\left\|\boldsymbol{q}-\boldsymbol{q}_{h}\right\|_{L^{\infty}\left(T ; L^{2}\left(\mathcal{T}_{h}\right)\right)} \leq C_{1}(1+T) h^{k+1},
$$

provided also that the projection $\left(\boldsymbol{P}_{\boldsymbol{V}}, P_{W}\right)$ used to define the initial condition has the following approximation property:

$$
\left\|\boldsymbol{q}^{0}-\boldsymbol{P}_{\boldsymbol{V}} \boldsymbol{q}^{0}\right\|_{L^{2}\left(\mathcal{T}_{h}\right)}+\left\|v^{0}-P_{W} v^{0}\right\|_{\left(L^{2}\left(\mathcal{T}_{h}\right)\right.} \leq C_{2} h^{k+1} .
$$

Moreover, if we take $\left(\boldsymbol{P}_{\boldsymbol{V}}, P_{W}\right):=\left(\boldsymbol{\Pi}_{\boldsymbol{V}}, \Pi_{W}\right)$, we have

$$
\begin{aligned}
& \left\|\left(v-v_{h}\right)_{t}\right\|_{L^{\infty}\left(T ; L^{2}\left(\mathcal{T}_{h}\right)\right)}+\left\|\left(\boldsymbol{q}-\boldsymbol{q}_{h}\right)_{t}\right\|_{L^{\infty}\left(T ; L^{2}\left(\mathcal{T}_{h}\right)\right)} \leq C_{3}(1+T) h^{k+1}, \\
& \left\|\boldsymbol{q}-\boldsymbol{q}_{h}^{\star}\right\|_{L^{\infty}\left(T ; L^{2}\left(\mathcal{T}_{h}\right)\right)}+\left\|\nabla \cdot\left(\boldsymbol{q}-\boldsymbol{q}_{h}^{\star}\right)\right\|_{L^{\infty}\left(T ; L^{2}(\Omega)\right)} \leq C_{4}(1+T) h^{k+1} .
\end{aligned}
$$

Finally, if, in addition, $k \geq 1$ and the elliptic regularity condition (2.9) holds, we have

$$
\left\|u-u_{h}^{\star}\right\|_{L^{\infty}\left(T ; L^{2}\left(\mathcal{T}_{h}\right)\right)} \leq C_{6}(1+T)^{2} h^{k+2} .
$$

The constants $C_{i}, i=1, \ldots, 6$ depend on $L^{\infty}(T ; B)$-norms of the exact solution $(\boldsymbol{q}, v)$, where $B$ is a space of the form $H^{\ell}\left(\mathcal{T}_{h}\right)$, the polynomial degree $k$, and the regularity constant of the elements but which are independent of the time $T$ and the maximum of the diameters of the elements of the mesh $h$.

Since we are using approximations of piecewise polynomials of degree $k \geq 0$ for the velocity and the flux, the optimal order of convergence for both approximations is clearly $k+1$. This result shows that the method reaches such optimal order of convergence uniformly in time. This result also shows that, for $k \geq 1$, the error in the postprocessed displacement $u_{h}^{\star}$ is of order $k+1$ for time $T$ of order $1 / \sqrt{h}$. This holds for unstructured, regular meshes. As pointed out in [11, where the first result of this type for hyperbolic problems was obtained for the transport equation in one space dimension (for the upwind DG method), this is one of the major advantages of using DG methods for wave propagation problems for long times $T$.

Let us also note that the estimates for the error of the approximation $u_{h}^{\star}$ were only proven whenever we take $\left(\boldsymbol{P}_{\boldsymbol{V}}, P_{W}\right):=\left(\boldsymbol{\Pi}_{\boldsymbol{V}}, \Pi_{W}\right)$ to define the initial condition and whenever the elliptic regularity inequality (2.9) holds. In practice, however, if we simply take $\left(\boldsymbol{P}_{\boldsymbol{V}}, P_{W}\right)$ to be the $L^{2}$-projection into the space $\boldsymbol{V}_{h} \times W_{h}$, the approximation $u_{h}^{\star}$ still converges with order $k+2$ for $k \geq 1$, even if the regularity inequality (2.9) does not hold, as the numerical experiments in [47] show. Our current technique does not allow us to account for these results.

\section{Proofs}

This section contains the proof of our convergence results. We begin by showing the existence and uniqueness of the approximate solution. We then prove the estimates of Theorem 2.2. First, we use an energy argument to obtain optimal convergence for the projection of the errors. Next, we use again an energy argument to obtain superconvergent estimates of the time derivatives of the projection of the 
errors. To achieve this, the initial condition has to be chosen in a special manner. Finally, we use a duality argument to obtain the last superconvergence estimate. The rest of the section is devoted to proving the estimates of the postprocessings.

Step 1: Existence and uniqueness of the approximate solution. Since the system defining the method (2.1) is square, we only need to show that the zero solution is the only solution whenever the initial condition, the boundary conditions, and the source term are all zero. The error estimates show that is is indeed the case.

Step 2: The equations of the projection of the errors. The projection of the errors satisfy equations similar to the ones for the approximate solutions in equations (2.1) as we see in the following result.

Lemma 3.1. We have

$$
\begin{aligned}
\left(c \boldsymbol{\varepsilon}_{t}^{q}, \boldsymbol{r}\right)_{\mathcal{T}_{h}}+\left(\varepsilon^{v}, \nabla \cdot \boldsymbol{r}\right)_{\mathcal{T}_{h}}-\left\langle\varepsilon^{\widehat{v}}, \boldsymbol{r} \cdot \boldsymbol{n}\right\rangle_{\partial \mathcal{T}_{h}} & =\left(c\left(\boldsymbol{\Pi}_{\boldsymbol{V}} \boldsymbol{q}_{t}-\boldsymbol{q}_{t}\right), \boldsymbol{r}\right)_{\mathcal{T}_{h}}, \\
\left(\rho \varepsilon_{t}^{v}, w\right)_{\mathcal{T}_{h}}+\left(\varepsilon^{q}, \nabla w\right)_{\mathcal{T}_{h}}-\left\langle\varepsilon^{\widehat{q}} \cdot \boldsymbol{n}, w\right\rangle_{\partial \mathcal{T}_{h}} & =\left(\rho\left(\Pi_{W} v_{t}-v_{t}\right), w\right)_{\mathcal{T}_{h}}, \\
\left\langle\varepsilon^{\widehat{q}} \cdot \boldsymbol{n}, \mu\right\rangle_{\partial \mathcal{T}_{h} \backslash \partial \Omega} & =0,
\end{aligned}
$$

for all $(\boldsymbol{r}, w, \mu) \in \mathbf{V}_{h} \times W_{h} \times M_{h}$ where

$$
\varepsilon^{\widehat{q}} \cdot \boldsymbol{n}=\varepsilon^{q} \cdot \boldsymbol{n}-\tau\left(\varepsilon^{v}-\varepsilon^{\widehat{v}}\right) \quad \text { on } \partial \mathcal{T}_{h} .
$$

Moreover,

$$
\begin{aligned}
\left\langle\varepsilon^{\widehat{v}}, \mu\right\rangle_{\partial \Omega_{D}} & =0, \\
\left\langle\varepsilon^{\widehat{q}} \cdot \boldsymbol{n}, \mu\right\rangle_{\partial \Omega_{N}} & =0,
\end{aligned}
$$

for any $\mu \in M_{h}$.

Proof. Since the exact solution $(\boldsymbol{q}, v)$ satisfies

$$
\begin{aligned}
& \left(c \boldsymbol{q}_{t}, \boldsymbol{r}\right)_{\mathcal{T}_{h}}+(v, \nabla \cdot \boldsymbol{r})_{\mathcal{T}_{h}}-\langle v, \boldsymbol{r} \cdot \boldsymbol{n}\rangle_{\partial \mathcal{T}_{h}}=0, \\
& \left(\rho v_{t}, w\right)_{\mathcal{T}_{h}}+(\boldsymbol{q}, \nabla w)_{\mathcal{T}_{h}}-\langle\boldsymbol{q} \cdot \boldsymbol{n}, w\rangle_{\partial \mathcal{T}_{h}}=(f, w)_{\mathcal{T}_{h}}
\end{aligned}
$$

for all $(\boldsymbol{r}, w, \mu) \in \mathbf{V}_{h} \times W_{h}$, we can use the first two orthogonality properties of the projection, (2.8a) and (2.8b), to obtain that

$$
\begin{aligned}
\left(c \boldsymbol{\Pi}_{\boldsymbol{V}} \boldsymbol{q}_{t}, \boldsymbol{r}\right)_{\mathcal{T}_{h}}+\left(\Pi_{W} v, \nabla \cdot \boldsymbol{r}\right)_{\mathcal{T}_{h}}-\langle v, \boldsymbol{r} \cdot \boldsymbol{n}\rangle_{\partial \mathcal{T}_{h}} & =\left(c\left(\boldsymbol{\Pi}_{\boldsymbol{V}} \boldsymbol{q}_{t}-\boldsymbol{q}_{t}\right), \boldsymbol{r}\right)_{\mathcal{T}_{h}}, \\
\left(\rho \Pi_{W} v_{t}, w\right)_{\mathcal{T}_{h}}+\left(\boldsymbol{\Pi}_{\boldsymbol{V}} \boldsymbol{q}, \nabla w\right)_{\mathcal{T}_{h}}-\left\langle\widehat{\Pi}_{\boldsymbol{V}} \boldsymbol{q} \cdot \boldsymbol{n}, w\right\rangle_{\partial \mathcal{T}_{h}} & =(f, w)_{\mathcal{T}_{h}}+\left(\rho\left(\Pi_{W} v_{t}-v_{t}\right), w\right)_{\mathcal{T}_{h}}
\end{aligned}
$$

for all $(\boldsymbol{r}, w, \mu) \in \mathbf{V}_{h} \times W_{h}$. Subtracting the first equation (2.1a) which defines the method from the first equation above, we obtain the first error equation (3.1a). Similarly, subtracting the second equation (2.1b) which defines the method from the second equation above, we obtain the second error equation (3.1b).

Let us show that the third error equation (3.1c) holds. By the definition of $\varepsilon^{\widehat{q}}$, for all $\mu \in M_{h}$,

$$
\begin{aligned}
\left\langle\varepsilon^{\widehat{q}} \cdot \boldsymbol{n}, \mu\right\rangle_{\partial \mathcal{T}_{h} \backslash \partial \Omega} & =\left\langle P_{M}(\boldsymbol{q} \cdot \boldsymbol{n})-\widehat{\boldsymbol{q}}_{h} \cdot \boldsymbol{n}, \mu\right\rangle_{\partial \mathcal{T}_{h} \backslash \partial \Omega} \\
& =\left\langle\boldsymbol{q} \cdot \boldsymbol{n}-\widehat{\boldsymbol{q}}_{h} \cdot \boldsymbol{n}, \mu\right\rangle_{\partial \mathcal{T}_{h} \backslash \partial \Omega},
\end{aligned}
$$

by the definition of $P_{M}$. Hence,

$$
\left\langle\varepsilon^{\widehat{q}} \cdot \boldsymbol{n}, \mu\right\rangle_{\partial \mathcal{T}_{h} \backslash \partial \Omega}=0 \quad \forall \mu \in M_{h},
$$


since the normal components of both $\boldsymbol{q}$ and $\widehat{\boldsymbol{q}}_{h}$ are single valued. This is the third error equation (3.1c).

Next, let us prove the identity (3.1d). By definition of $\varepsilon^{\widehat{q}},(2.2)$,

$$
\varepsilon^{\widehat{q}} \cdot \boldsymbol{n}=P_{M}(\boldsymbol{q} \cdot \boldsymbol{n})-\widehat{\boldsymbol{q}}_{h} \cdot \boldsymbol{n}=P_{M}(\boldsymbol{q} \cdot \boldsymbol{n})-\boldsymbol{q}_{h} \cdot \boldsymbol{n}+\tau\left(v_{h}-\widehat{v}_{h}\right),
$$

by the definition of the numerical trace $\widehat{\boldsymbol{q}}_{h}$, (2.2). By the third orthogonality property of the projection, (2.8c),

$$
\varepsilon^{\widehat{q}} \cdot \boldsymbol{n}=\left\langle\left(\Pi_{\boldsymbol{V}} \boldsymbol{q}-\boldsymbol{q}_{h}\right) \cdot \boldsymbol{n}-\tau\left(\Pi_{W} v-v_{h}-P_{M} v+\widehat{v}_{h}\right)=\boldsymbol{\varepsilon}^{q} \cdot \boldsymbol{n}-\tau\left(\varepsilon^{v}-\varepsilon^{\widehat{v}}\right),\right.
$$

by the definition of the projections of the errors.

Let us now show that the errors $\varepsilon^{\widehat{v}}$ and $\varepsilon^{\widehat{q}}$ satisfy (3.2). By definition of $\varepsilon^{\widehat{v}}$, for all $\mu \in M_{h}$,

$$
\left\langle\varepsilon^{\widehat{v}}, \mu\right\rangle_{\partial \Omega_{D}}=\left\langle P_{M} v-\widehat{v}_{h}, \mu\right\rangle_{\partial \Omega_{D}}=\left\langle v-\widehat{v}_{h}, \mu\right\rangle_{\partial \Omega_{D}}=0
$$

by the definition of the projection $P_{M}$ and by the boundary conditions for the exact solution and for the approximate solution in (2.4a). Similarly, for all $\mu \in M_{h}$,

$$
\left\langle\varepsilon^{\widehat{q}} \cdot \boldsymbol{n}, \mu\right\rangle_{\partial \Omega_{D}}=\left\langle P_{M}(\boldsymbol{q} \cdot \boldsymbol{n})-\widehat{\boldsymbol{q}}_{h} \cdot \boldsymbol{n}, \mu\right\rangle_{\partial \Omega_{D}}=\left\langle\boldsymbol{q} \cdot \boldsymbol{n}-\widehat{\boldsymbol{q}}_{h} \cdot \boldsymbol{n}, \mu\right\rangle_{\partial \Omega_{D}}=0,
$$

by the definition of the projection $P_{M}$ and by the boundary conditions for the exact solution and for the approximate solution in (2.4b). This completes the proof.

Step 3: Estimate of the projection of the errors. An energy argument for the projection of the errors enables us to obtain optimal convergence for the method. We begin by obtaining the following energy identity.

Lemma 3.2. For any $t>0$, we have that

$$
\begin{aligned}
& \left\|\sqrt{c} \varepsilon^{q}(t)\right\|_{L^{2}\left(\mathcal{T}_{h}\right)}^{2}+\left\|\sqrt{\rho} \varepsilon^{v}(t)\right\|_{L^{2}\left(\mathcal{T}_{h}\right)}^{2}+2 \int_{0}^{t}\left\|\sqrt{\tau}\left(\varepsilon^{v}-\varepsilon^{\widehat{v}}\right)\right\|_{L^{2}\left(\partial \mathcal{T}_{h}\right)}^{2} \\
& =\left\|\sqrt{c} \varepsilon^{q}(0)\right\|_{L^{2}\left(\mathcal{T}_{h}\right)}^{2}+\left\|\sqrt{\rho} \varepsilon^{v}(0)\right\|_{L^{2}\left(\mathcal{T}_{h}\right)}^{2} \\
& \quad+2 \int_{0}^{t}\left(c\left(\Pi_{\boldsymbol{V}} \boldsymbol{q}_{t}-\boldsymbol{q}_{t}\right), \boldsymbol{\varepsilon}^{q}\right)_{\mathcal{T}_{h}}+2 \int_{0}^{t}\left(\rho\left(\Pi_{W} v_{t}-v_{t}\right), \varepsilon^{v}\right) .
\end{aligned}
$$

Proof. Taking $\boldsymbol{r}:=\varepsilon^{q}$ in the first error equation of Lemma 3.1, $w:=\varepsilon^{v}$ in the second, $\mu:=\varepsilon^{\widehat{v}}$ in the third and sixth, and $\mu:=-\varepsilon^{\widehat{q}} \cdot \boldsymbol{n}$ in the fifth, and adding them up, we obtain, after simple algebraic manipulations, that

$$
\begin{aligned}
\frac{1}{2} \frac{d}{d t}\left\|\sqrt{c} \varepsilon^{q}\right\|_{L^{2}\left(\mathcal{T}_{h}\right)}^{2}+ & \frac{1}{2} \frac{d}{d t}\left\|\sqrt{\rho} \varepsilon^{v}\right\|_{L^{2}\left(\mathcal{T}_{h}\right)}^{2}-\left\langle\left(\varepsilon^{\widehat{q}}-\varepsilon^{q}\right) \cdot \boldsymbol{n},\left(\varepsilon^{v}-\varepsilon^{\widehat{v}}\right)\right\rangle_{\partial T_{h}} \\
& =\left(c\left(\boldsymbol{\Pi}_{\boldsymbol{V}} \boldsymbol{q}_{t}-\boldsymbol{q}_{t}\right), \boldsymbol{\varepsilon}^{q}\right)_{\mathcal{T}_{h}}+\left(\rho\left(\Pi_{W} v_{t}-v_{t}\right), \varepsilon^{v}\right)
\end{aligned}
$$

The result follows after using the expression for $\varepsilon^{\widehat{q}}$ in Lemma 3.1 and after integrating in time. This completes the proof. 
We are now ready to prove the first estimate of Theorem 2.2. By the CauchySchwarz inequality, we get

$$
\begin{aligned}
& \left\|\sqrt{c} \varepsilon^{q}(t)\right\|_{L^{2}\left(\mathcal{T}_{h}\right)}^{2}+\left\|\sqrt{\rho} \varepsilon^{v}(t)\right\|_{L^{2}\left(\mathcal{T}_{h}\right)}^{2}+2 \int_{0}^{t}\left\|\sqrt{\tau}\left(\varepsilon^{v}-\varepsilon^{\widehat{v}}\right)\right\|_{L^{2}\left(\partial \mathcal{T}_{h}\right)}^{2} \\
& \leq\left\|\sqrt{c} \varepsilon^{q}(0)\right\|_{L^{2}\left(\mathcal{T}_{h}\right)}^{2}+\left\|\sqrt{\rho} \varepsilon^{v}(0)\right\|_{L^{2}\left(\mathcal{T}_{h}\right)}^{2} \\
& \quad+2 \int_{0}^{t}\left\|\sqrt{c}\left(\Pi_{\boldsymbol{V}} \boldsymbol{q}_{t}-\boldsymbol{q}_{t}\right)\right\|_{L^{2}\left(\mathcal{T}_{h}\right)}\left\|\sqrt{c} \varepsilon^{q}\right\|_{L^{2}\left(\mathcal{T}_{h}\right)} \\
& \quad+2 \int_{0}^{t}\left\|\sqrt{\rho}\left(\Pi_{W} v_{t}-v_{t}\right)\right\|_{L^{2}\left(\mathcal{T}_{h}\right)}\left\|\sqrt{\rho} \varepsilon^{v}\right\|_{L^{2}\left(\mathcal{T}_{h}\right)} .
\end{aligned}
$$

We now follow an argument similar to Grönwall's; see for instance [61, p. 33]. Hence, if we call the right-hand side of the above inequality $\chi(t)$, we obtain

$$
\begin{aligned}
\frac{d}{d t} \chi(t) & =2\left\|\sqrt{c}\left(\boldsymbol{\Pi}_{\boldsymbol{V}} \boldsymbol{q}_{t}-\boldsymbol{q}_{t}\right)\right\|_{L^{2}\left(\mathcal{T}_{h}\right)}\left\|\sqrt{c} \varepsilon^{q}\right\|_{L^{2}\left(\mathcal{T}_{h}\right)} \\
& +2\left\|\sqrt{\rho}\left(\Pi_{W} v_{t}-v_{t}\right)\right\|_{L^{2}\left(\mathcal{T}_{h}\right)}\left\|\sqrt{\rho} \varepsilon^{v}\right\|_{L^{2}\left(\mathcal{T}_{h}\right)}, \\
& \leq\left(2\left\|\sqrt{c}\left(\boldsymbol{\Pi}_{\boldsymbol{V}} \boldsymbol{q}_{t}-\boldsymbol{q}_{t}\right)\right\|_{L^{2}\left(\mathcal{T}_{h}\right)}+2\left\|\sqrt{\rho}\left(\Pi_{W} v_{t}-v_{t}\right)\right\|_{L^{2}\left(\mathcal{T}_{h}\right)}\right) \sqrt{\chi(t)} .
\end{aligned}
$$

Solving for $\chi(t)$ yields

$$
\sqrt{\chi(t)} \leq \sqrt{\chi(0)}+\int_{0}^{t}\left(\left\|\sqrt{c}\left(\boldsymbol{\Pi}_{\boldsymbol{V}} \boldsymbol{q}_{t}-\boldsymbol{q}_{t}\right)\right\|_{L^{2}\left(\mathcal{T}_{h}\right)}+\left\|\sqrt{\rho}\left(\Pi_{W} v_{t}-v_{t}\right)\right\|_{L^{2}\left(\mathcal{T}_{h}\right)}\right),
$$

and the result follows. This concludes the proof of the first estimate of Theorem 2.2 .

Using the same argument, we also get an energy inequality of the time derivative of the projection of the errors.

Corollary 3.1. We have

$$
\begin{aligned}
& {\left[\left\|\sqrt{c} \varepsilon_{t}^{q}\right\|_{L^{\infty}\left(T ; L^{2}\left(\mathcal{T}_{h}\right)\right)}^{2}+\left\|\sqrt{\rho} \varepsilon_{t}^{v}\right\|_{L^{\infty}\left(T ; L^{2}\left(\mathcal{T}_{h}\right)\right)}^{2}+2\left\|\sqrt{\tau}\left(\varepsilon_{t}^{v}-\varepsilon_{t}^{\widehat{v}}\right)\right\|_{L^{2}\left(T ; L^{2}\left(\partial \mathcal{T}_{h}\right)\right)}^{2}\right]^{1 / 2}} \\
& \quad \leq\left[\left\|\sqrt{c} \varepsilon_{t}^{q}(0)\right\|_{L^{2}\left(\mathcal{T}_{h}\right)}^{2}+\left\|\sqrt{\rho} \varepsilon_{t}^{v}(0)\right\|_{L^{2}\left(\mathcal{T}_{h}\right)}^{2}\right]^{1 / 2} \\
& \quad+\left\|\sqrt{c}\left(\boldsymbol{\Pi}_{\boldsymbol{V}} \boldsymbol{q}_{t t}-\boldsymbol{q}_{t t}\right)\right\|_{L^{1}\left(T ; L^{2}\left(\mathcal{T}_{h}\right)\right)}+\left\|\sqrt{\rho}\left(\Pi_{W} v_{t t}-v_{t t}\right)\right\|_{L^{1}\left(T ; L^{2}\left(\mathcal{T}_{h}\right)\right)} .
\end{aligned}
$$

Step 4: Estimate of the time derivative of the projections of the errors. Next, we obtain the second estimate of Theorem 2.2 by using an energy argument and by exploiting the fact that we are taking a special projection to define the initial condition.

We begin with the energy argument obtained in Corollary 3.1 However, we see that we still need to estimate the time derivative of the projection of the errors at the initial time.

Lemma 3.3. Suppose that we take $\left(\boldsymbol{P}_{\boldsymbol{V}}, P_{W}\right):=\left(\boldsymbol{\Pi}_{\boldsymbol{V}}, \Pi_{W}\right)$ as the projection with which we define the initial condition. Then, we have

$$
\begin{aligned}
& \left\|\sqrt{c} \varepsilon_{t}^{q}(0)\right\|_{L^{2}\left(\mathcal{T}_{h}\right)}^{2}+\left\|\sqrt{\rho} \varepsilon_{t}^{v}(0)\right\|_{L^{2}\left(\mathcal{T}_{h}\right)}^{2} \\
& \quad \leq\left[\left\|\sqrt{c}\left(\boldsymbol{\Pi}_{\boldsymbol{V}} \boldsymbol{q}_{t}-\boldsymbol{q}_{t}\right)(0)\right\|_{L^{2}\left(\mathcal{T}_{h}\right)}^{2}+\left\|\sqrt{\rho}\left(\Pi_{W} v_{t}-v_{t}\right)(0)\right\|_{L^{2}\left(\mathcal{T}_{h}\right)}^{2}\right] .
\end{aligned}
$$


Proof. Consider the two first error equations (3.1a) and (3.1b), namely,

$$
\begin{gathered}
\left(c\left(\boldsymbol{q}-\boldsymbol{q}_{h}\right)_{t}(0), \boldsymbol{r}\right)_{\mathcal{T}_{h}}+\left(\varepsilon^{v}(0), \nabla \cdot \boldsymbol{r}\right)_{\mathcal{T}_{h}}-\left\langle\varepsilon^{\widehat{v}}(0), \boldsymbol{r} \cdot \boldsymbol{n}\right\rangle_{\partial \mathcal{T}_{h}}=0, \\
\left(\rho\left(v-v_{h}\right)_{t}(0), w\right)_{\mathcal{T}_{h}}+\left(\varepsilon^{q}(0), \nabla w\right)_{\mathcal{T}_{h}}-\left\langle\varepsilon^{\widehat{q}}(0) \cdot \boldsymbol{n}, w\right\rangle_{\partial \mathcal{T}_{h}}=0 .
\end{gathered}
$$

We note that, since $\left(\boldsymbol{P}_{\boldsymbol{V}}, P_{W}\right):=\left(\boldsymbol{\Pi}_{\boldsymbol{V}}, \Pi_{W}\right)$, we have that $\varepsilon^{v}(0)=0$ and that $\varepsilon^{q}(0)=\mathbf{0}$, so

$$
\begin{gathered}
\left(c\left(\boldsymbol{q}-\boldsymbol{q}_{h}\right)_{t}(0), \boldsymbol{r}\right)_{\mathcal{T}_{h}}-\left\langle\varepsilon^{\widehat{v}}(0), \boldsymbol{r} \cdot \boldsymbol{n}\right\rangle_{\partial \mathcal{T}_{h}}=0, \\
\left(\rho\left(v-v_{h}\right)_{t}(0), w\right)_{\mathcal{T}_{h}}-\left\langle\varepsilon^{\widehat{q}}(0) \cdot \boldsymbol{n}, w\right\rangle_{\partial \mathcal{T}_{h}}=0 .
\end{gathered}
$$

We then take $\boldsymbol{r}=\varepsilon_{t}^{q}(0)$ and $w=\varepsilon_{t}^{v}(0)$, add the two equations together, and insert the HDG projection to get

$$
\begin{aligned}
& \left\|\sqrt{c} \varepsilon_{t}^{q}(0)\right\|_{L^{2}(\Omega)}^{2}+\left\|\sqrt{\rho} \varepsilon_{t}^{v}(0)\right\|_{L^{2}(\Omega)}^{2}+\left\langle\varepsilon^{\widehat{v}}(0), \varepsilon_{t}^{q}(0) \cdot \boldsymbol{n}\right\rangle_{\partial \mathcal{T}_{h}}+\left\langle\varepsilon^{\widehat{q}}(0) \cdot \boldsymbol{n}, \varepsilon_{t}^{v}(0),\right\rangle_{\partial \mathcal{T}_{h}} \\
& =\left(c\left(\boldsymbol{\Pi}_{\boldsymbol{V}} \boldsymbol{q}_{t}-\boldsymbol{q}_{t}\right)(0), \boldsymbol{\varepsilon}_{t}^{q}(0)\right)_{\Omega}+\left(\rho\left(\Pi_{W} v_{t}-v_{t}\right)(0), \varepsilon_{t}^{v}(0)\right)_{\Omega} .
\end{aligned}
$$

We now show that the two boundary terms disappear to get

$$
\begin{aligned}
& \left\|\sqrt{c} \varepsilon_{t}^{q}(0)\right\|_{L^{2}(\Omega)}^{2}+\left\|\sqrt{\rho} \varepsilon_{t}^{v}(0)\right\|_{L^{2}(\Omega)}^{2} \\
& =\left(c\left(\boldsymbol{\Pi}_{\boldsymbol{V}} \boldsymbol{q}_{t}-\boldsymbol{q}_{t}\right)(0), \boldsymbol{\varepsilon}_{t}^{q}(0)\right)_{\Omega}+\left(\rho\left(\Pi_{W} v_{t}-v_{t}\right)(0), \varepsilon_{t}^{v}(0)\right)_{\Omega} .
\end{aligned}
$$

We turn to the first boundary term. By the third error equation (3.1c) and the boundary conditions (3.2), we have that

$$
\begin{aligned}
0 & =\left\langle\varepsilon^{\widehat{q}}(0) \cdot \boldsymbol{n}, \mu\right\rangle_{\partial \mathcal{T}_{h} \backslash \Omega_{D}}+\left\langle\tau \varepsilon^{\widehat{v}}(0), \mu\right\rangle_{\partial \Omega_{D}} \\
& =\left\langle\varepsilon^{q}(0) \cdot \boldsymbol{n}-\tau\left(\varepsilon^{v}(0)-\varepsilon^{\widehat{v}}(0)\right), \mu\right\rangle_{\partial \mathcal{T}_{h} \backslash \Omega_{D}}+\left\langle\tau \varepsilon^{\widehat{v}}(0), \mu\right\rangle_{\partial \Omega_{D}} \\
& =\left\langle\tau \varepsilon^{\widehat{v}}(0), \mu\right\rangle_{\partial \mathcal{T}_{h}} \quad \forall \mu \in M_{h},
\end{aligned}
$$

using that $\varepsilon^{v}(0)=0$ and $\varepsilon^{q}(0)=\mathbf{0}$. Taking $\mu=\left(\tau^{+}+\tau^{-}\right) \varepsilon^{\widehat{v}}(0)$ on each edge, where $\tau^{-}=0$ for edges lying on the boundary, we see that $\left(\tau^{+}+\tau^{-}\right) \varepsilon^{\widehat{v}}(0)=0$ on $\partial \mathcal{T}_{h}$. This implies that, on $\partial \mathcal{T}_{h}^{+}$, which we define to be the part of $\partial \mathcal{T}_{h}$ where $\tau^{+}+\tau^{-}>0$, we must have $\varepsilon^{\widehat{v}}(0)=0$. Hence we have that

$$
\left\langle\varepsilon^{\widehat{v}}(0), \mu\right\rangle_{\partial \mathcal{T}_{h}}=\left\langle\varepsilon^{\widehat{v}}(0), \mu\right\rangle_{\partial \mathcal{T}_{h} \backslash \mathcal{T}_{h}^{+}} .
$$

On the other hand, $\tau^{+}+\tau^{-}=0$ on $\partial \mathcal{T}_{h} \backslash \mathcal{T}_{h}^{+}$and, since $\tau$ is positive, $\tau^{+}=$ $\tau^{-}=0$ therein. As a consequence, the normal component of $\varepsilon^{q}=\varepsilon^{\widehat{q}}$ is single valued on $\partial \mathcal{T}_{h} \backslash \mathcal{T}_{h}^{+}$, and so is $\varepsilon_{t}^{q}$. With $\mu=\varepsilon_{t}^{q}(0) \cdot \boldsymbol{n}$, we thus see that $\left\langle\varepsilon^{\widehat{v}}(0), \boldsymbol{\varepsilon}_{t}^{q}(0) \cdot \boldsymbol{n}\right\rangle_{\partial \mathcal{T}_{h} \backslash \partial \mathcal{T}_{h}^{+} \backslash \partial \Omega}=0$. Using the boundary conditions (3.2a) and $(3.2 \mathrm{~b})$, the later derived in time, we then have that $\left\langle\varepsilon^{\widehat{v}}(0), \varepsilon_{t}^{q}(0) \cdot \boldsymbol{n}\right\rangle_{\partial \mathcal{T}_{h} \backslash \partial \mathcal{T}_{h}^{+}}=$ $\left\langle\varepsilon^{\widehat{v}}(0), \varepsilon_{t}^{\widehat{q}}(0) \cdot \boldsymbol{n}\right\rangle_{\partial \mathcal{T}_{h} \backslash \partial \mathcal{T}_{h}^{+}}=0$.

We now turn to the second boundary term. We have that

$$
\varepsilon^{\widehat{q}}(0) \cdot \boldsymbol{n}=\varepsilon^{q}(0) \cdot \boldsymbol{n}-\tau\left(\varepsilon^{v}(0)-\varepsilon^{\widehat{v}}(0)\right)=\tau \varepsilon^{\widehat{v}}(0)=0,
$$

from the previous argument. Therefore, in particular, $\left\langle\varepsilon^{\widehat{q}}(0) \cdot \boldsymbol{n}, \varepsilon_{t}^{v}(0)\right\rangle_{\partial \mathcal{T}_{h}}=0$.

The estimate follows after applying Cauchy-Schwarz and Young's inequalities to equation 3.3 This concludes the proof.

The second estimate of Theorem 2.2 follows by inserting the estimate of the last lemma into the estimate of the previous one. 
Step 5: The dual problem. Here, we introduce a dual problem whose solution we are going to use to obtain the remaining estimate of Theorem 2.2. We define the function $\varsigma$ to be the solution of the acoustic wave equation

$$
\rho \varsigma_{t t}-\nabla \cdot(A \nabla \varsigma)=0,
$$

with final and boundary conditions

$$
\begin{aligned}
\varsigma(T, x) & =0, & \varsigma_{t}(T, x) & =\theta(x), \\
\varsigma\left(t, y_{D}\right) & =0, & \boldsymbol{n} \cdot A \nabla \varsigma\left(t, y_{N}\right) & =0,
\end{aligned}
$$

for any $x \in \Omega, y_{D} \in \partial \Omega_{D}, y_{N} \in \partial \Omega_{N}$, and $t \in[0, T)$.

Proposition 3.1. There is a constant $C$ depending on $\rho$ and $A$ such that

$$
\|\varsigma\|_{L^{\infty}\left(T ; H^{1}(\Omega)\right)}+\left\|\varsigma_{t}\right\|_{L^{\infty}\left(T ; L^{2}(\Omega)\right)} \leq C\|\theta\|_{L^{2}(\Omega)} .
$$

Moreover, if the elliptic regularity (2.9) holds, then

$$
\|\underline{s}\|_{L^{\infty}\left(T ; H^{2}(\Omega)\right)} \leq C C_{\mathrm{reg}}\|\theta\|_{L^{2}(\Omega)},
$$

where $\underline{\varsigma}(t):=\int_{t}^{T} \varsigma$.

Proof. The first inequality is a direct consequence of the conservation of the energy. Let us prove the second inequality. To do that, we begin by noting that we can integrate in time from $t$ to $T$ the equation defining the dual problem (3.4a) to get that $\rho\left(\varsigma_{t}(T)-\varsigma_{t}(t)\right)-\nabla \cdot(A \nabla \underline{\varsigma})=0$. Using the fact $\rho \varsigma_{t}(T)=\theta$, by the final condition (3.4b), and noting that $-\varsigma_{t}(t)=\underline{\varsigma}_{t t}$, we see that $\underline{\varsigma}$ satisfies the acoustic wave equation

with final and boundary conditions

$$
\rho \underline{\varsigma}_{t t}-\nabla \cdot(A \nabla \underline{\varsigma})=-\rho \theta
$$

$$
\begin{aligned}
\underline{\varsigma}(T, x) & =0, & \underline{\varsigma}_{t}(T, x) & =0, \\
\underline{\varsigma}\left(t, y_{D}\right) & =0, & \boldsymbol{n} \cdot A \nabla_{\underline{\varsigma}}\left(t, y_{N}\right) & =0,
\end{aligned}
$$

for any $x \in \Omega, y_{D} \in \partial \Omega_{D}, y_{N} \in \partial \Omega_{N}$, and $t \in[0, T)$.

Next, we use the elliptic regularity inequality (2.9) on $\varsigma$ to get

$$
\begin{aligned}
\|\underline{\varsigma}\|_{H^{2}(\Omega)} & \leq C_{\mathrm{reg}}\|\nabla \cdot A \nabla \underline{\varsigma}\|_{L^{2}(\Omega)} \\
& \leq C_{\mathrm{reg}}\left(\left\|\underline{\varsigma}_{t t}\right\|_{L^{2}(\Omega)}+\left\|\underline{\varsigma}_{t t}-\nabla \cdot A \nabla \underline{\varsigma}\right\|_{L^{2}(\Omega)}\right) \\
& \leq C_{\mathrm{reg}}\left(\left\|\varsigma_{t}\right\|_{L^{2}(\Omega)}+\|\rho \theta\|_{L^{2}(\Omega)}\right),
\end{aligned}
$$

and the result follows from the first inequality. This completes the proof.

Step 6: A duality argument for $\mathrm{P}_{l-1} \overline{\varepsilon^{v}}(T)$. To estimate $\mathrm{P}_{l-1} \overline{\varepsilon^{v}}(T)$ for $1 \leq$ $l \leq k$, we use the identity

$$
\left\|\rho \mathrm{P}_{l-1} \overline{\varepsilon^{v}}(T)\right\|_{L^{2}(\Omega)}=\sup _{\theta \in C_{0}^{\infty}(\Omega)} \frac{\left(\mathrm{P}_{l-1} \overline{\varepsilon^{v}}(T), \rho \theta\right)_{\Omega}}{\|\theta\|_{L^{2}(\Omega)}},
$$

and we find an expression for the numerator of the right-hand side of this identity.

Lemma 3.4. Suppose that $k \geq 1$. Then, for any $\theta \in C_{0}^{\infty}(\Omega)$, we have

$$
\begin{aligned}
\left(\mathrm{P}_{l-1} \overline{\varepsilon^{v}}(T), \rho \theta\right)_{\Omega}= & \left(\varepsilon^{v}(0), \mathrm{P}_{l-1} \rho \underline{\underline{s}_{t}}(0)\right)_{\Omega}+\int_{0}^{T}\left(\rho\left(v_{h}-v\right)_{t}, \rho^{-1} \mathrm{P}_{l-1} \rho \underline{\varsigma_{t}}-I_{h} \underline{\varsigma_{t}}\right)_{\Omega} \\
& -\left(\varepsilon^{q}(0), \nabla I_{h} \underline{\underline{\varsigma_{t}}}(0)\right)_{\Omega}+\int_{0}^{T}\left(c\left(\boldsymbol{q}_{h}-\boldsymbol{q}\right)_{t}, \boldsymbol{\Pi}_{l}^{\mathrm{BDM}} \underline{A \nabla \varsigma}+A \nabla I_{h} \underline{\underline{\varsigma_{t}}}\right)_{\Omega},
\end{aligned}
$$


where $I_{h}$ is any interpolant from $L^{2}(\Omega)$ into $W_{h} \cap H_{0, D}^{1}(\Omega)$, and $H_{0, D}^{1}(\Omega)$ denotes the space of functions in $H^{1}(\Omega)$ with null trace on $\partial \Omega_{D}$.

Proof. We start by using the final condition of the dual problem, $\varsigma_{t}(T):=\varsigma_{t}(T)=\theta$ (see $(3.4 \mathrm{~b})$ ) and the fact that $\overline{\varepsilon^{v}}(0)=0$ to get

$$
\begin{aligned}
\Theta & :=\left(\mathrm{P}_{l-1} \overline{\varepsilon^{v}}(T), \rho \theta\right)_{\Omega}=\left(\mathrm{P}_{l-1} \overline{\varepsilon^{v}}(T), \rho \varsigma_{t}(T)\right)_{\Omega} \\
& =\int_{0}^{T}\left[\left(\mathrm{P}_{l-1} \varepsilon^{v}, \rho \varsigma_{t}\right)_{\Omega}+\left(\mathrm{P}_{l-1} \overline{\varepsilon^{v}}, \rho \varsigma_{t t}\right)_{\Omega}\right] \\
& =\int_{0}^{T}\left[\left(\mathrm{P}_{l-1} \varepsilon^{v}, \rho \varsigma_{t}\right)_{\Omega}+\left(\mathrm{P}_{l-1} \overline{\varepsilon^{v}}, \nabla \cdot A \nabla \varsigma\right)_{\Omega}\right]
\end{aligned}
$$

since $\rho \varsigma_{t t}=\nabla \cdot A \nabla \varsigma$, by the equation (3.4a) defining the dual problem. If we now use the identity $\int_{0}^{T} f(t) g(t) d t=f(0) \underline{g}(0)+\int_{0}^{T} f_{t}(t) \underline{g}(t) d t$, where $\underline{g}(t):=\int_{t}^{T} g(s) d s$, we get

$$
\begin{aligned}
\Theta & =\left(\mathrm{P}_{l-1} \varepsilon^{v}(0), \rho \underline{\varsigma_{t}}(0)\right)_{\Omega}+\int_{0}^{T}\left[\left(\mathrm{P}_{l-1} \varepsilon_{t}^{v}, \rho \underline{\varsigma_{t}}\right)_{\Omega}+\left(\mathrm{P}_{l-1} \varepsilon^{v}, \nabla \cdot A \nabla \underline{\varsigma}\right)_{\Omega}\right] \\
& =\left(\varepsilon^{v}(0), \mathrm{P}_{l-1} \rho \underline{\varsigma_{t}}(0)\right)_{\Omega}+\int_{0}^{T}\left[\left(\varepsilon_{t}^{v}, \mathrm{P}_{l-1} \rho \underline{\varsigma_{t}}\right)_{\Omega}+\left(\varepsilon^{v}, \mathrm{P}_{l-1} \nabla \cdot A \nabla \underline{\varsigma}\right)_{\Omega}\right],
\end{aligned}
$$

by the definition of the projection $\mathrm{P}_{l-1}$.

Let us work on the term $\theta:=\left(\varepsilon^{v}, \mathrm{P}_{l-1} \nabla \cdot A \nabla \underline{\varsigma}\right)_{\Omega}$. By the well-known commutativity property of the BDM projection $\mathrm{P}_{l-1} \nabla \cdot=\nabla \cdot \boldsymbol{\Pi}_{l}^{\mathrm{BDM}}$ (see [35]), we get

$$
\theta=\left(\varepsilon^{v}, \nabla \cdot \boldsymbol{\Pi}_{l}^{\mathrm{BDM}} A \nabla \underline{\varsigma}\right)_{\Omega} .
$$

By the first equation for the projection of the errors, (3.1a), with $\boldsymbol{r}:=\mathbf{\Pi}_{l}^{\mathrm{BDM}} A \nabla \underline{\varsigma}$, we obtain

$$
\begin{aligned}
\theta=\left(c\left(\boldsymbol{q}_{h}-\boldsymbol{q}\right)_{t}, \boldsymbol{\Pi}_{l}^{\mathrm{BDM}} A \nabla \underline{\varsigma}\right)_{\Omega}+\left\langle\varepsilon^{\widehat{v}}, \boldsymbol{\Pi}_{l}^{\mathrm{BDM}} A \nabla_{\underline{\varsigma}} \cdot \boldsymbol{n}\right\rangle_{\partial \mathcal{T}_{h}} \\
=\left(c\left(\boldsymbol{q}_{h}-\boldsymbol{q}\right)_{t}, \boldsymbol{\Pi}_{l}^{\mathrm{BDM}} A \nabla \underline{\varsigma}\right)_{\Omega}
\end{aligned}
$$

since $\left\langle\varepsilon^{\widehat{v}}, \boldsymbol{\Pi}_{l}^{\mathrm{BDM}} A \nabla \underline{\varsigma} \cdot \boldsymbol{n}\right\rangle_{\partial \mathcal{T}_{h}}=0$ because $\varepsilon^{\widehat{v}}$ is single valued and vanishes on the boundary $\partial \Omega_{D}$ (see (3.2a) ), and because $\boldsymbol{\Pi}_{l}^{\mathrm{BDM}} A \nabla \underline{\varsigma} \in H(\mathrm{div})$, and $A \nabla \underline{\varsigma} \cdot \boldsymbol{n}$ vanishes on the boundary $\partial \Omega_{N}$ (see $(\underline{3.4 \mathrm{C}})$ ).

We add and subtract the term $\nabla I_{h} \underline{\underline{\varsigma_{t}}}$ as follows:

$$
\begin{aligned}
\theta & =\left(\left(\boldsymbol{q}_{h}-\boldsymbol{q}\right)_{t}, c \boldsymbol{\Pi}_{l}^{\mathrm{BDM}} A \nabla \underline{\underline{\varsigma}}+\nabla I_{h} \underline{\underline{\varsigma_{t}}}\right)_{\Omega}-\left(\left(\boldsymbol{q}_{h}-\boldsymbol{q}\right)_{t}, \nabla I_{h} \underline{\underline{\varsigma_{t}}}\right)_{\Omega} \\
& =\left(\left(\boldsymbol{q}_{h}-\boldsymbol{q}\right)_{t}, c \boldsymbol{\Pi}_{l}^{\mathrm{BDM}} A \nabla \underline{\underline{\varsigma}}+\nabla I_{h} \underline{\underline{\varsigma_{t}}}\right)_{\Omega}+\left(\varepsilon_{t}^{q}, \nabla I_{h} \underline{\underline{\varsigma_{t}}}\right)_{\Omega},
\end{aligned}
$$

by the orthogonality property (2.8a) of the projection $\boldsymbol{\Pi}_{\boldsymbol{V}}$. If we now derive with respect to time the second error equation (3.1b) and take $w:=I_{h} \underline{\underline{S_{t}}}$, we get that

$$
\begin{aligned}
\theta= & \left(\left(\boldsymbol{q}_{h}-\boldsymbol{q}\right)_{t}, c \boldsymbol{\Pi}_{l}^{\mathrm{BDM}} A \nabla_{\underline{\varsigma}}+\nabla I_{h} \underline{\underline{\varsigma_{t}}}\right)_{\Omega}+\left(\left(v_{h}-v\right)_{t t}, \rho I_{h} \underline{\underline{\varsigma_{t}}}\right)_{\Omega}+\left\langle\varepsilon_{t}^{\widehat{q}} \cdot \boldsymbol{n}, I_{h} \underline{\underline{\varsigma_{t}}}\right\rangle_{\partial \mathcal{T}_{h}} \\
& =\left(\left(\boldsymbol{q}_{h}-\boldsymbol{q}\right)_{t}, c \boldsymbol{\Pi}_{l}^{\mathrm{BDM}} A \nabla \underline{\underline{\varsigma}}+\nabla I_{h} \underline{\underline{\varsigma_{t}}}\right)_{\Omega}+\left(\left(v_{h}-v\right)_{t t}, \rho I_{h} \underline{\underline{\varsigma_{t}}}\right)_{\Omega},
\end{aligned}
$$

since, after deriving with respect to time the error equation (3.1c) and setting $\mu:=I_{h} \underline{\underline{\varsigma_{t}}}$, we see that $\left\langle\varepsilon_{t}^{\widehat{q}} \cdot \boldsymbol{n}, I_{h} \underline{\underline{\varsigma_{t}}}\right\rangle_{\partial \mathcal{T}_{h}}=\left\langle\varepsilon_{t}^{\widehat{q}} \cdot \boldsymbol{n}, I_{h} \underline{\underline{\varsigma_{t}}}\right\rangle_{\partial \Omega}=\left\langle\varepsilon_{t}^{\widehat{q}} \cdot \boldsymbol{n}, I_{h} \underline{\underline{\varsigma_{t}}}\right\rangle_{\partial \Omega_{D}}=0$, by the Neumann boundary condition (3.4c), and since, by construction, $I_{h} \underline{\underline{\varsigma_{t}}}=0$ on $[0, T] \times \partial \Omega_{D}$. 
Inserting the above expression for $\theta$ into the last expression for $\Theta$ and rearranging terms, we get

$$
\begin{aligned}
\Theta= & \left(\varepsilon^{v}(0), \mathrm{P}_{l-1} \rho \underline{\varsigma_{t}}(0)\right)_{\Omega}+\int_{0}^{T}\left(\left(v_{h}-v\right)_{t t}, \rho I_{h} \underline{\underline{\varsigma_{t}}}\right)_{\Omega} \\
& +\int_{0}^{T}\left(\varepsilon_{t}^{v}, \mathrm{P}_{l-1} \rho \underline{\underline{\varsigma}_{t}}\right)_{\Omega}+\int_{0}^{T}\left(\left(\boldsymbol{q}_{h}-\boldsymbol{q}\right)_{t}, c \boldsymbol{\Pi}_{l}^{\mathrm{BDM}} A \nabla \underline{\varsigma}+\nabla I_{h} \underline{\underline{\varsigma_{t}}}\right)_{\Omega} \\
= & \left(\varepsilon^{v}(0), \mathrm{P}_{l-1} \rho \underline{\varsigma_{t}}(0)\right)_{\Omega}-\left(\rho\left(v_{h}-v\right)_{t}(0), I_{h} \underline{\underline{\varsigma_{t}}}(0)\right)_{\Omega}-\int_{0}^{T}\left(\left(v_{h}-v\right)_{t}, \rho I_{h} \underline{\varsigma_{t}}\right)_{\Omega} \\
& +\int_{0}^{T}\left(\varepsilon_{t}^{v}, \mathrm{P}_{l-1} \rho \underline{\varsigma_{t}}\right)_{\Omega}+\int_{0}^{T}\left(\left(\boldsymbol{q}_{h}-\boldsymbol{q}\right)_{t}, c \boldsymbol{\Pi}_{l}^{\mathrm{BDM}} A \nabla \underline{\varsigma}+\nabla I_{h} \underline{\underline{\varsigma_{t}}}\right)_{\Omega} \\
= & \left(\varepsilon^{v}(0), \mathrm{P}_{l-1} \rho \underline{\varsigma_{t}}(0)\right)_{\Omega}-\left(\rho\left(v_{h}-v\right)_{t}(0), I_{h} \underline{\underline{\varsigma_{t}}}(0)\right)_{\Omega} \\
& +\int_{0}^{T}\left(\left(v_{h}-v\right)_{t}, \mathrm{P}_{l-1} \rho \underline{\varsigma_{t}}-\rho I_{h} \underline{\varsigma_{t}}\right)_{\Omega}+\int_{0}^{T}\left(\left(\boldsymbol{q}_{h}-\boldsymbol{q}\right)_{t}, c \boldsymbol{\Pi}_{l}^{\mathrm{BDM}} A \nabla \underline{\varsigma}+\nabla I_{h} \underline{\underline{\varsigma_{t}}}\right)_{\Omega}
\end{aligned}
$$

by the orthogonality property of $\Pi_{W},(2.8 \mathrm{~b})$. The result follows by taking $w:=$ $I_{h} \underline{\underline{\varsigma_{t}}}(0)$ in the second error equation (3.1b) and reasoning as above. This completes the proof of Lemma 3.4 .

Step 7: Estimate of $\rho \mathrm{P}_{l-1} \overline{\varepsilon^{v}}(T)$. A direct consequence of the identity of Lemma 3.4 is the following result.

Lemma 3.5. Suppose that $k \geq 1$ and that we take $\left(\boldsymbol{P}_{\boldsymbol{V}}, P_{W}\right):=\left(\boldsymbol{\Pi}_{\boldsymbol{V}}, \Pi_{W}\right)$ as the projection with which we define the initial condition. Assume that the elliptic regularity (2.9) inequality holds. Then,

$$
\begin{aligned}
\left\|\rho \mathrm{P}_{l-1} \overline{\varepsilon^{v}}(T)\right\|_{L^{2}\left(\mathcal{T}_{h}\right)} \leq C T h( & \left\|\sqrt{\rho}\left(v-v_{h}\right)_{t}\right\|_{L^{\infty}\left(T ; L^{2}\left(\mathcal{T}_{h}\right)\right)} \\
& \left.+\left\|\sqrt{c}\left(\boldsymbol{q}-\boldsymbol{q}_{h}\right)_{t}\right\|_{L^{\infty}\left(T ; L^{2}\left(\mathcal{T}_{h}\right)\right)}\right) .
\end{aligned}
$$

Proof. If we take $\left(\boldsymbol{P}_{\boldsymbol{V}}, P_{W}\right):=\left(\boldsymbol{\Pi}_{\boldsymbol{V}}, \Pi_{W}\right)$ as the projection with which we define the initial conditions, the identity of Lemma 3.4 gives us that

$$
\begin{aligned}
\left(\mathrm{P}_{l-1} \overline{\varepsilon^{v}}(T), \rho \theta\right)_{\Omega}= & \int_{0}^{T}\left(\left(v_{h}-v\right)_{t}, \mathrm{P}_{l-1} \rho \underline{\varsigma_{t}}-\rho I_{h} \underline{\varsigma_{t}}\right)_{\Omega} \\
& +\int_{0}^{T}\left(\left(\boldsymbol{q}_{h}-\boldsymbol{q}\right)_{t}, c \boldsymbol{\Pi}_{l}^{\mathrm{BDM}} A \nabla \underline{\varsigma}+\nabla I_{h \underline{\varsigma_{t}}}\right)_{\Omega} .
\end{aligned}
$$

After a straightforward application of the Cauchy-Schwarz inequality, we obtain that the quantity $\left(\mathrm{P}_{l-1} \overline{\varepsilon^{v}}(T), \rho \theta\right)_{\Omega}$ is bounded by

$$
\begin{aligned}
T & \left(\left\|\left(v_{h}-v\right)_{t}\right\|_{L^{\infty}\left(T ; L^{2}\left(\mathcal{T}_{h}\right)\right)}\left\|\rho \underline{\varsigma_{t}}-\mathrm{P}_{l-1} \rho \underline{\varsigma_{t}}\right\|_{L^{\infty}\left(T ; L^{2}\left(\mathcal{T}_{h}\right)\right)}\right. \\
& +\left\|\sqrt{\rho}\left(v_{h}-v\right)_{t}\right\|_{L^{\infty}\left(T ; L^{2}\left(\mathcal{T}_{h}\right)\right)}\left\|\sqrt{\rho}\left(\underline{\varsigma_{t}}-I_{h} \underline{\varsigma_{t}}\right)\right\|_{L^{\infty}\left(T ; L^{2}\left(\mathcal{T}_{h}\right)\right)} \\
& +\left\|\sqrt{c}\left(\boldsymbol{q}_{h}-\boldsymbol{q}\right)_{t}\right\|_{L^{\infty}\left(T ; L^{2}\left(\mathcal{T}_{h}\right)\right)}\left\|\sqrt{c}\left(\boldsymbol{\Pi}_{l}^{\mathrm{BDM}} A \nabla \underline{\varsigma}-A \nabla \underline{\varsigma}\right)\right\|_{L^{\infty}\left(T ; L^{2}\left(\mathcal{T}_{h}\right)\right)} \\
& \left.+\left\|\left(\boldsymbol{q}_{h}-\boldsymbol{q}\right)_{t}\right\|_{L^{\infty}\left(T ; L^{2}\left(\mathcal{T}_{h}\right)\right)}\left\|\nabla \underline{\underline{\varsigma_{t}}}-\nabla I_{h} \underline{\underline{\varsigma_{t}}}\right\|_{L^{\infty}\left(T ; L^{2}\left(\mathcal{T}_{h}\right)\right)}\right) .
\end{aligned}
$$

If we now use the well-known approximation properties of the projections $\boldsymbol{\Pi}_{l}^{\mathrm{BDM}}$, $P_{W}$, and $\mathrm{P}_{l-1}$, we get that

$$
\begin{aligned}
\left\|\rho \underline{\varsigma_{t}}-\mathrm{P}_{l-1} \rho \underline{\varsigma_{t}}\right\|_{L^{\infty}\left(T ; L^{2}(\Omega)\right)} & \leq C h\left|\rho \underline{\varsigma_{t}}\right|_{L^{\infty}\left(T ; H^{1}(\Omega)\right)} \leq C h\left\|_{\varsigma}\right\|_{L^{\infty}\left(T ; H^{1}(\Omega)\right)}, \\
\left\|\underline{\varsigma_{t}}-I_{h} \underline{\varsigma_{t}}\right\|_{L^{\infty}\left(T ; L^{2}(\Omega)\right)} & \leq C h{\underline{\underline{t}_{t}}}_{L^{\infty}\left(T ; H^{1}(\Omega)\right)}=\left.C h\right|_{L^{\infty}\left(T ; H^{1}(\Omega)\right) .}
\end{aligned}
$$


We can bound the right-hand side of these two inequalities by $C h\|\theta\|_{L^{2}(\Omega)}$ using Proposition 3.1. In addition,

$$
\begin{aligned}
& \left\|\boldsymbol{\Pi}_{l}^{\mathrm{BDM}} A \nabla \underline{\varsigma}-A \nabla \underline{\varsigma}\right\|_{L^{\infty}\left(T ; L^{2}(\Omega)\right)} \leq C h|A \nabla \underline{\varsigma}|_{L^{\infty}\left(T ; H^{1}(\Omega)\right)}=\left.\left.C h\right|_{\underline{\varsigma}}\right|_{L^{\infty}\left(T ; H^{2}(\Omega)\right)}, \\
& \left\|\nabla I_{h} \underline{\underline{S_{t}}}-\nabla \underline{\underline{S_{t}}}\right\|_{L^{\infty}\left(T ; L^{2}(\Omega)\right)} \leq\left.\left. C h\right|_{\underline{\underline{S_{t}}}}\right|_{L^{\infty}\left(T ; H^{2}(\Omega)\right)}=\left.\left.C h\right|_{\underline{\underline{S}}}\right|_{L^{\infty}\left(T ; H^{2}(\Omega)\right)},
\end{aligned}
$$

for $l \geq 0$ and $k \geq 1$. If the elliptic regularity inequality (2.9) holds, we can again bound the right-hand side of these two inequalities by $C h\|\theta\|_{L^{2}(\Omega)}$ using Proposition 3.1 .

We can now use the identity (3.5) for the $L^{2}$-norm of $\rho \mathrm{P}_{l-1} \varepsilon^{v}(T)$ to obtain the estimate. This completes the proof.

We can now use these results to prove the last inequality of Theorem 2.2. Indeed, since

$$
\left\|\mathrm{P}_{l-1} \overline{\varepsilon^{v}}(T)\right\|_{L^{2}\left(\mathcal{T}_{h}\right)} \leq C\left\|\rho \mathrm{P}_{l-1} \overline{\varepsilon^{v}}(T)\right\|_{L^{2}\left(\mathcal{T}_{h}\right)},
$$

we obtain the wanted inequality by using the second estimate of Theorem 2.2 . This completes the proof of Theorem 2.2 .

Step 8: The error in $\boldsymbol{q}_{h}^{\star}$. Let us now prove the error estimates for $\boldsymbol{q}_{h}^{\star}$ in Proposition 2.1. By definition of $\boldsymbol{q}_{h}^{\star}$, 2.5), and by definition of the Raviart-Thomas projection, $\Pi_{k}^{\mathrm{RT}}$, we have that $\boldsymbol{\delta}:=\Pi_{k}^{\mathrm{RT}} \boldsymbol{q}-\boldsymbol{q}_{h}^{\star}$ is the only element of $\left(\mathcal{P}_{k}(K)\right)^{d}+\boldsymbol{x} \mathcal{P}_{k}(K)$ satisfying

$$
\begin{aligned}
(\boldsymbol{\delta}, \boldsymbol{v})_{K} & =\left(\boldsymbol{q}-\boldsymbol{q}_{h}, \boldsymbol{v}\right)_{K} & & \forall \boldsymbol{v} \in\left(\mathcal{P}_{k-1}(K)\right)^{d}, \\
\langle\boldsymbol{\delta} \cdot \boldsymbol{n}, \mu\rangle_{F} & =\left\langle\left(\boldsymbol{q}-\widehat{\boldsymbol{q}}_{h}\right) \cdot \boldsymbol{n}, \mu\right\rangle_{F} & & \forall \mu \in \mathcal{P}_{k}(F) \text { and all faces } F \text { of } K .
\end{aligned}
$$

Using the definitions of $\varepsilon^{q}$ and $\varepsilon^{\widehat{q}}$, we get that

$$
\begin{aligned}
(\boldsymbol{\delta}, \boldsymbol{v})_{K} & =\left(\varepsilon^{q}, \boldsymbol{v}\right)_{K} & & \forall \boldsymbol{v} \in\left(\mathcal{P}_{k-1}(K)\right)^{d}, \\
\langle\boldsymbol{\delta} \cdot \boldsymbol{n}, \mu\rangle_{F} & =\left\langle\varepsilon^{q} \cdot \boldsymbol{n}-\tau\left(\varepsilon^{v}-\varepsilon^{\widehat{v}}\right), \mu\right\rangle_{F} & & \forall \mu \in \mathcal{P}_{k}(F) \text { and all faces } F \text { of } K .
\end{aligned}
$$

The first estimate of Proposition 2.1 is a direct consequence of the above equations.

Now, if we use the definition of $\boldsymbol{q}_{h}^{\star}$ in the second equation defining the HDG method, (2.1b), we obtain that

$$
\left(\rho\left(v_{h}\right)_{t}, w\right)_{K}-\left(\nabla \cdot \boldsymbol{q}_{h}^{\star}, w\right)_{K}=(f, w)_{K} \quad \forall w \in \mathcal{P}_{k}(K),
$$

and so

$$
(\nabla \cdot \boldsymbol{\delta}, w)_{K}=\left(\rho\left(v-v_{h}\right)_{t}, w\right)_{K} \quad \forall w \in \mathcal{P}_{k}(K) .
$$

The second estimate of Proposition 2.1 immediately follows by taking $w:=\nabla \cdot \boldsymbol{\delta}$.

Step 9: The error in $u_{h}^{\star}$. In this step, we obtain the last estimate of Proposition 2.1. To do that, we begin with the following result.

Lemma 3.6. Set $\varepsilon^{u}:=\mathrm{P}_{k+1} u-u_{h}^{\star}$. For any element $K \in \mathcal{T}_{h}$, we have that

$$
\left\|\varepsilon^{u}\right\|_{L^{2}(K)} \leq\left\|\mathrm{P}_{0} \varepsilon^{u}\right\|_{L^{2}(K)}+C h\left(\left\|\nabla\left(u-\mathrm{P}_{k+1} u\right)\right\|_{L^{2}(K)}+\left\|c\left(\boldsymbol{q}_{h}-\boldsymbol{q}\right)\right\|_{L^{2}(K)}\right) .
$$

Proof. We have that

$$
\begin{aligned}
\left\|\varepsilon^{u}\right\|_{L^{2}(K)} & \leq\left\|\mathrm{P}_{0} \varepsilon^{u}\right\|_{L^{2}(K)}+\left\|\mathrm{P}_{0} \varepsilon^{u}-\varepsilon^{u}\right\|_{L^{2}(K)} \\
& \leq\left\|\mathrm{P}_{0} \varepsilon^{u}\right\|_{L^{2}(K)}+C h\left\|\nabla \varepsilon^{u}\right\|_{L^{2}(K)} .
\end{aligned}
$$


It remains to estimate $\left\|\nabla \varepsilon^{u}\right\|_{L^{2}(K)}$. To do that, we proceed as follows. By the definition of $\varepsilon^{u}$, we have that, for any $w \in \mathrm{P}_{k+1}(K)$,

$$
\begin{aligned}
\left(\nabla \varepsilon^{u}, \nabla w\right)_{K} & =\left(\nabla\left(\mathrm{P}_{k+1} u-u_{h}^{\star}\right), \nabla w\right)_{K} \\
& =\left(\nabla\left(\mathrm{P}_{k+1} u-u\right), \nabla w\right)_{K}+\left(\nabla\left(u-u_{h}^{\star}\right), \nabla w\right)_{K} \\
& =\left(\nabla\left(\mathrm{P}_{k+1} u-u\right), \nabla w\right)_{K}+\left(c\left(\boldsymbol{q}-\boldsymbol{q}_{h}\right), \nabla w\right)_{K},
\end{aligned}
$$

by the first equation defining $u_{h}^{\star}$, (2.7a). Taking $w:=\varepsilon^{u}$ and using the CauchySchwarz inequality, we get that

$$
\left\|\nabla \varepsilon^{u}\right\|_{L^{2}(K)} \leq\left\|\nabla\left(u-\mathrm{P}_{k+1} u\right)\right\|_{L^{2}(K)}+\left\|c\left(\boldsymbol{q}_{h}-\boldsymbol{q}\right)\right\|_{L^{2}(K)},
$$

and the result follows. This completes the proof.

Now, we obtain an estimate of $\left\|\mathrm{P}_{0} \varepsilon^{u}\right\|_{L^{2}(K)}$.

Lemma 3.7. If $k \geq 1$, we have that $\left\|\mathrm{P}_{0} \varepsilon^{u}\right\|_{L^{2}(K)} \leq\left\|\mathrm{P}_{0} \overline{\varepsilon^{v}}\right\|_{L^{2}(K)}$.

Proof. We proceed as follows. We have that

$$
\left\|\mathrm{P}_{0} \varepsilon^{u}\right\|_{L^{2}(K)}^{2}=\left(\mathrm{P}_{0} \varepsilon^{u}, \mathrm{P}_{0} \varepsilon^{u}\right)_{K}=\left(\mathrm{P}_{k+1} u-u_{h}^{\star}, \mathrm{P}_{0} \varepsilon^{u}\right)_{K}=\left(u-u_{h}, \mathrm{P}_{0} \varepsilon^{u}\right)_{K}
$$

by the second equation defining $u_{h}^{\star},\left(2.7 \mathrm{~b}\right.$ ). However, by the definition of $u_{h},(2.6)$, and since $u=u_{0}+\bar{v}$, we have that

$$
\left\|\mathrm{P}_{0} \varepsilon^{u}\right\|_{L^{2}(K)}^{2}=\left(u(0)-u_{h}(0), \mathrm{P}_{0} \varepsilon^{u}\right)_{K}+\left(\bar{v}-\bar{v}_{h}, \mathrm{P}_{0} \varepsilon^{u}\right)_{K}=\left(\bar{v}-\bar{v}_{h}, \mathrm{P}_{0} \varepsilon^{u}\right)_{K}
$$

by the second equation defining $u_{h}(0),(2.6 \mathrm{~b})$. Now, since $k \geq 1$, we can use the orthogonality property of the projection $\Pi_{W},(2.8 \mathrm{~b})$, to obtain that

$$
\left\|\mathrm{P}_{0} \varepsilon^{u}\right\|_{L^{2}(K)}^{2}=\left(\Pi_{W} \bar{v}-\bar{v}_{h}, \mathrm{P}_{0} \varepsilon^{u}\right)_{K}=\left(\overline{\varepsilon^{v}}, \mathrm{P}_{0} \varepsilon^{u}\right)_{K}=\left(\mathrm{P}_{0} \overline{\varepsilon^{v}}, \mathrm{P}_{0} \varepsilon^{u}\right)_{K},
$$

and the result follows. This completes the proof.

It is clear that by inserting the estimate of the last lemma into the inequality of the previous one, we obtain the last inequality of Proposition 2.1.

\section{EXTENSIONS AND CONCLUDING REMARKS}

Let us briefly sketch how to extend the results of this paper to other HDG and mixed methods. Note that Cockburn, Qiu, and Shi 21 gave general sufficient conditions on the finite element spaces for the superconvergence of a wide variety of HDG and mixed methods for second-order elliptic equations. The same conditions are also sufficient to obtain the superconvergence of those methods for the acoustic wave equation. Only minor modifications have to be made to extend our error analysis to this general case. In fact, we only have to replace the auxiliary projections $\mathrm{P}_{l-1}$ and $\boldsymbol{\Pi}_{l}^{\mathrm{BDM}}$, used in Steps 6 and 7 , by suitably defined projections. Let us discuss how.

Let us begin by noting that the only properties we are using of these projections are the following:

(i) $\mathrm{P}_{l-1}: \boldsymbol{H}^{1}(\Omega) \mapsto W_{h}$,

(ii) $\boldsymbol{\Pi}_{l}^{\mathrm{BDM}}: \boldsymbol{H}^{1}(\Omega) \mapsto H(\Omega, \operatorname{div}) \cap \boldsymbol{V}_{h}$,

(iii) $\mathrm{P}_{l-1} \nabla \cdot=\nabla \cdot \boldsymbol{\Pi}_{l}^{\mathrm{BDM}}$,

(iv) $\left\|\boldsymbol{\Pi}_{l}^{\mathrm{BDM}} \boldsymbol{\zeta}-\boldsymbol{\zeta}\right\|_{L^{2}(\Omega)} \leq C h|\boldsymbol{\zeta}|_{H^{1}(\Omega)}$. 
Next, we note that we only need to ensure the superconvergence of $\mathrm{P}_{l-1} \overline{\varepsilon^{v}}$ in the case in which $\mathrm{P}_{l-1}=\mathrm{P}_{0}$, as we see in Theorem 2.2 and in Proposition 2.1. Thus, for all the methods considered in [21, we can keep $\boldsymbol{\Pi}_{1}^{\mathrm{BDM}}$ when using simplexes. When the elements $K \in \mathcal{T}_{h}$ are squares or cubes, we can use the projection $\boldsymbol{\Pi}_{[0]}^{\mathrm{RT}}$ which, on each element, is the well-known projection into the lowest index Raviart-Thomas space $\mathrm{RT}_{[0]}(K)$. When the elements are prisms, we can use the projection $\boldsymbol{\Pi}_{\langle 0\rangle}^{\mathrm{RT}}$ which, on each element, is the projection into the lowest index Raviart-Thomas space $\mathrm{RT}_{\langle 0\rangle}(K)$.

Let us note that, for the mixed methods considered in [21, namely, the wellknown RT, BDFM, and BDM methods (on simplexes, squares, cubes, or prisms) and the newly found TNT elements (on squares and cubes), we can replace the projections $\mathrm{P}_{l-1}$ and $\boldsymbol{\Pi}_{l}^{\mathrm{BDM}}$ by the projections $\Pi_{W}$ and $\boldsymbol{\Pi}_{\boldsymbol{V}}$, respectively. Moreover, in this case, we also have that $\boldsymbol{q}_{h}^{\star}=\boldsymbol{q}_{h}$.

Let us end by pointing out that the extension of our results to the fully discrete case and to elastodynamics constitutes the subject of ongoing research.

\section{REFERENCES}

[1] R. M. Alford, K. R. Kelly, and D. M. Boore, Accuracy of finite-difference modeling of the acoustic wave equation, Geophysics 39 (1974), no. 6, 834-842.

[2] Garth A. Baker, Error estimates for finite element methods for second order hyperbolic equations, SIAM J. Numer. Anal. 13 (1976), no. 4, 564-576. MR0423836 (54 \#11810)

[3] Peter Bastian and Béatrice Rivière, Superconvergence and H(div) projection for discontinuous Galerkin methods, Internat. J. Numer. Methods Fluids 42 (2003), no. 10, 1043-1057, DOI 10.1002/fld.562. MR1991232 (2004f:65177)

[4] E. Bécache, P. Joly, and C. Tsogka, An analysis of new mixed finite elements for the approximation of wave propagation problems, SIAM J. Numer. Anal. 37 (2000), no. 4, 1053-1084 (electronic), DOI 10.1137/S0036142998345499. MR1756415(2001d:65124)

[5] Oscar P. Bruno, New high-order integral methods in computational electromagnetism, CMES Comput. Model. Eng. Sci. 5 (2004), no. 4, 319-330. MR2070392

[6] Oscar P. Bruno and Michael C. Haslam, Efficient high-order evaluation of scattering by periodic surfaces: vector-parametric gratings and geometric singularities, Waves Random Complex Media 20 (2010), no. 4, 530-550, DOI 10.1080/17455030.2010.499151. MR2747419 (2011k:65189)

[7] Fatih Celiker, Bernardo Cockburn, and Ke Shi, A projection-based error analysis of HDG methods for Timoshenko beams, Math. Comp. 81 (2012), no. 277, 131-151, DOI 10.1090/S0025-5718-2011-02522-6. MR2833490(2012h:65143)

[8] Fatih Celiker, Bernardo Cockburn, and Ke Shi, Hybridizable discontinuous Galerkin methods for Timoshenko beams, J. Sci. Comput. 44 (2010), no. 1, 1-37, DOI 10.1007/s10915-0109357-2. MR2647497 (2011f:65249)

[9] Brandon Chabaud and Bernardo Cockburn, Uniform-in-time superconvergence of HDG methods for the heat equation, Math. Comp. 81 (2012), no. 277, 107-129, DOI 10.1090/S00255718-2011-02525-1. MR2833489(2012i:65198)

[10] Yanlai Chen and Bernardo Cockburn, Analysis of variable-degree HDG methods for convection-diffusion equations. Part I: general nonconforming meshes, IMA J. Numer. Anal. 32 (2012), no. 4, 1267-1293, DOI 10.1093/imanum/drr058. MR2991828

[11] Yingda Cheng and Chi-Wang Shu, Superconvergence of discontinuous Galerkin and local discontinuous Galerkin schemes for linear hyperbolic and convection-diffusion equations in one space dimension, SIAM J. Numer. Anal. 47 (2010), no. 6, 4044-4072, DOI 10.1137/090747701. MR:2585178 (2011e:65187)

[12] Bernardo Cockburn and Jintao Cui, An analysis of HDG methods for the vorticity-velocitypressure formulation of the Stokes problem in three dimensions, Math. Comp. 81 (2012), no. 279, 1355-1368, DOI 10.1090/S0025-5718-2011-02575-5. MR2904582 
[13] Bernardo Cockburn, Bo Dong, and Johnny Guzmán, A superconvergent LDG-hybridizable Galerkin method for second-order elliptic problems, Math. Comp. 77 (2008), no. 264, 18871916, DOI 10.1090/S0025-5718-08-02123-6. MR2429868 (2009d:65166)

[14] Bernardo Cockburn, Bo Dong, and Johnny Guzmán, A hybridizable and superconvergent discontinuous Galerkin method for biharmonic problems, J. Sci. Comput. 40 (2009), no. 1-3, 141-187, DOI 10.1007/s10915-009-9279-z. MR2511731(2010h:65223)

[15] Bernardo Cockburn, Jayadeep Gopalakrishnan, and Raytcho Lazarov, Unified hybridization of discontinuous Galerkin, mixed, and continuous Galerkin methods for second order elliptic problems, SIAM J. Numer. Anal. 47 (2009), no. 2, 1319-1365, DOI 10.1137/070706616. MR2485455(2010b:65251)

[16] Bernardo Cockburn, Jayadeep Gopalakrishnan, Ngoc Cuong Nguyen, Jaume Peraire, and Francisco-Javier Sayas, Analysis of HDG methods for Stokes flow, Math. Comp. 80 (2011), no. 274, 723-760, DOI 10.1090/S0025-5718-2010-02410-X. MR2772094 (2012d:65273)

[17] Bernardo Cockburn, Jayadeep Gopalakrishnan, and Francisco-Javier Sayas, A projectionbased error analysis of HDG methods, Math. Comp. 79 (2010), no. 271, 1351-1367, DOI 10.1090/S0025-5718-10-02334-3. MR2629996 (2011d:65354)

[18] Bernardo Cockburn, Johnny Guzmán, and Haiying Wang, Superconvergent discontinuous Galerkin methods for second-order elliptic problems, Math. Comp. 78 (2009), no. 265, 1-24, DOI 10.1090/S0025-5718-08-02146-7. MR2448694 (2009i:65213)

[19] Bernardo Cockburn, Guido Kanschat, and Dominik Schotzau, A locally conservative LDG method for the incompressible Navier-Stokes equations, Math. Comp. 74 (2005), no. 251, 1067-1095 (electronic), DOI 10.1090/S0025-5718-04-01718-1. MR2136994 (2006a:65157)

[20] B. Cockburn, N. C. Nguyen, and J. Peraire, A comparison of HDG methods for Stokes flow, J. Sci. Comput. 45 (2010), no. 1-3, 215-237, DOI 10.1007/s10915-010-9359-0. MR2679797 (2011g:65246)

[21] Bernardo Cockburn, Weifeng Qiu, and Ke Shi, Conditions for superconvergence of HDG methods for second-order elliptic problems, Math. Comp. 81 (2012), no. 279, 1327-1353, DOI 10.1090/S0025-5718-2011-02550-0. MR2904581

[22] B. Cockburn and F.J. Sayas, Divergence-conforming HDG methods for Stokes flow, submitted.

[23] Bernardo Cockburn and Ke Shi, Conditions for superconvergence of HDG methods for Stokes flow, Math. Comp. 82 (2013), no. 282, 651-671, DOI 10.1090/S0025-5718-2012-02644-5. MR.3008833

[24] Ramon Codina, Finite element approximation of the hyperbolic wave equation in mixed form, Comput. Methods Appl. Mech. Engrg. 197 (2008), no. 13-16, 1305-1322, DOI 10.1016/j.cma.2007.11.006. MR.2387006 (2009a:65248)

[25] Gary Cohen and Patrick Joly, Construction analysis of fourth-order finite difference schemes for the acoustic wave equation in nonhomogeneous media, SIAM J. Numer. Anal. 33 (1996), no. 4, 1266-1302, DOI 10.1137/S0036142993246445. MR.1403546 (97k:65234)

[26] David Colton and Rainer Kress, Inverse acoustic and electromagnetic scattering theory, 2nd ed., Applied Mathematical Sciences, vol. 93, Springer-Verlag, Berlin, 1998. MR1635980 (99c:35181)

[27] Lawrence C. Cowsar, Todd F. Dupont, and Mary F. Wheeler, A priori estimates for mixed finite element methods for the wave equation, Comput. Methods Appl. Mech. Engrg. 82 (1990), no. 1-3, 205-222, DOI 10.1016/0045-7825(90)90165-I. Reliability in computational mechanics (Austin, TX, 1989). MR1077657 (91i:65163)

[28] Lawrence C. Cowsar, Todd F. Dupont, and Mary F. Wheeler, A priori estimates for mixed finite element approximations of second-order hyperbolic equations with absorbing boundary conditions, SIAM J. Numer. Anal. 33 (1996), no. 2, 492-504, DOI 10.1137/0733026. MR,1388485 (96m:65093)

[29] M. A. Dablain, The application of high-order differencing to the scalar wave equation, Geophysics 51 (1986), no. 1, 54-66.

[30] Todd Dupont, L2-estimates for Galerkin methods for second order hyperbolic equations, SIAM J. Numer. Anal. 10 (1973), 880-889. MR0349045 (50 \#1539)

[31] Alexandre Ern and Jean-Luc Guermond, Theory and practice of finite elements, Applied Mathematical Sciences, vol. 159, Springer-Verlag, New York, 2004. MR2050138(2005d:65002)

[32] Lawrence C. Evans, Partial differential equations, Graduate Studies in Mathematics, vol. 19, American Mathematical Society, Providence, RI, 1998. MR.1625845 (99e:35001) 
[33] Abdelaâziz Ezziani and Patrick Joly, Local time stepping and discontinuous Galerkin methods for symmetric first order hyperbolic systems, J. Comput. Appl. Math. 234 (2010), no. 6, 1886-1895, DOI 10.1016/j.cam.2009.08.094. MR2644184(2011h:65172)

[34] Richard S. Falk and Gerard R. Richter, Explicit finite element methods for symmetric hyperbolic equations, SIAM J. Numer. Anal. 36 (1999), no. 3, 935-952 (electronic), DOI 10.1137/S0036142997329463. MR.1688992 (2000b:65181)

[35] Franco Brezzi and Michel Fortin, Mixed and hybrid finite element methods, Springer Series in Computational Mathematics, vol. 15, Springer-Verlag, New York, 1991. MR.1115205 (92d:65187)

[36] Lucia Gastaldi and Ricardo H. Nochetto, Sharp maximum norm error estimates for general mixed finite element approximations to second order elliptic equations, RAIRO Modél. Math. Anal. Numér. 23 (1989), no. 1, 103-128 (English, with French summary). MR.1015921 (91b:65125)

[37] Tunc Geveci, On the application of mixed finite element methods to the wave equations, RAIRO Modél. Math. Anal. Numér. 22 (1988), no. 2, 243-250 (English, with French summary). MR 945124 (89i:65116)

[38] L. Greengard and V. Rokhlin, A fast algorithm for particle simulations, J. Comput. Phys. 73 (1987), no. 2, 325-348, DOI 10.1016/0021-9991(87)90140-9. MR918448 (88k:82007)

[39] Marcus J. Grote, Anna Schneebeli, and Dominik Schötzau, Discontinuous Galerkin finite element method for the wave equation, SIAM J. Numer. Anal. 44 (2006), no. 6, 2408-2431 (electronic), DOI 10.1137/05063194X. MR2272600(2007k:65149)

[40] Eleanor W. Jenkins, Numerical solution of the acoustic wave equation using RaviartThomas elements, J. Comput. Appl. Math. 206 (2007), no. 1, 420-431, DOI 10.1016/j.cam.2006.08.003. MR.2337454 (2008e:76113)

[41] Eleanor W. Jenkins, Béatrice Rivière, and Mary F. Wheeler, A priori error estimates for mixed finite element approximations of the acoustic wave equation, SIAM J. Numer. Anal. 40 (2002), no. 5, 1698-1715 (electronic), DOI 10.1137/S0036142901388068. MR.1950619 (2003k:65120)

[42] Heinz-Otto Kreiss, N. Anders Petersson, and Jacob Yström, Difference approximations of the Neumann problem for the second order wave equation, SIAM J. Numer. Anal. 42 (2004), no. 3, 1292-1323 (electronic), DOI 10.1137/S003614290342827X. MR2113686 (2005j:65085)

[43] Timo Lähivaara, Matti Malinen, Jari P. Kaipio, and Tomi Huttunen, Computational aspects of the discontinuous Galerkin method for the wave equation, J. Comput. Acoust. 16 (2008), no. 4, 507-530, DOI 10.1142/S0218396X08003762. MR2523080 (2010e:65162)

[44] Yang Liu and Mrinal K. Sen, A new time-space domain high-order finite-difference method for the acoustic wave equation, J. Comput. Phys. 228 (2009), no. 23, 8779-8806, DOI 10.1016/j.jcp.2009.08.027. MR 2558777 (2010m:65182)

[45] Peter Monk and Gerard R. Richter, A discontinuous Galerkin method for linear symmetric hyperbolic systems in inhomogeneous media, J. Sci. Comput. 22/23 (2005), 443-477, DOI 10.1007/s10915-004-4132-5. MR 2142205 (2006b:65144)

[46] N. C. Nguyen, J. Peraire, and B. Cockburn, A hybridizable discontinuous Galerkin method for the incompressible Navier-Stokes equations (AIAA Paper 2010-362), Proceedings of the 48th AIAA Aerospace Sciences Meeting and Exhibit (Orlando, Florida), January 2010.

[47] N. C. Nguyen, J. Peraire, and B. Cockburn, High-order implicit hybridizable discontinuous Galerkin methods for acoustics and elastodynamics, J. Comput. Phys. 230 (2011), no. 10, 3695-3718, DOI 10.1016/j.jcp.2011.01.035. MR2783813 (2012g:65206)

[48] N. C. Nguyen, J. Peraire, and B. Cockburn, An implicit high-order hybridizable discontinuous Galerkin method for the incompressible Navier-Stokes equations, J. Comput. Phys. 230 (2011), no. 4, 1147-1170, DOI 10.1016/j.jcp.2010.10.032. MR2753354 (2011k:76055)

[49] N. C. Nguyen, J. Peraire, and B. Cockburn, Hybridizable discontinuous Galerkin methods, Proceedings of the International Conference on Spectral and High Order Methods (Trondheim, Norway), Lect. Notes Comput. Sci. Engrg., Springer Verlag, June 2009.

[50] N. C. Nguyen, J. Peraire, and B. Cockburn, An implicit high-order hybridizable discontinuous Galerkin method for linear convection-diffusion equations, J. Comput. Phys. 228 (2009), no. 9, 3232-3254, DOI 10.1016/j.jcp.2009.01.030. MR2513831(2010h:65232)

[51] N. C. Nguyen, J. Peraire, and B. Cockburn, An implicit high-order hybridizable discontinuous Galerkin method for nonlinear convection-diffusion equations, J. Comput. Phys. 228 (2009), no. 23, 8841-8855, DOI 10.1016/j.jcp.2009.08.030. MR2558780(2011a:65411) 
[52] N. C. Nguyen, J. Peraire, and B. Cockburn, A hybridizable discontinuous Galerkin method for Stokes flow, Comput. Methods Appl. Mech. Engrg. 199 (2010), no. 9-12, 582-597, DOI 10.1016/j.cma.2009.10.007. MR2796169 (2011m:65286)

[53] N. Nishimura, Fast multipole accelerated boundary integral equation methods, Applied Mechanics Reviews 55 (2002), no. 4, 299-324.

[54] J. Peraire, N. C. Nguyen, and B. Cockburn, A hybridizable discontinuous Galerkin method for the compressible Euler and Navier-Stokes equations (AIAA Paper 2010-363), Proceedings of the 48th AIAA Aerospace Sciences Meeting and Exhibit (Orlando, Florida), January 2010.

[55] Steffen Petersen, Charbel Farhat, and Radek Tezaur, A space-time discontinuous Galerkin method for the solution of the wave equation in the time domain, Internat. J. Numer. Methods Engrg. 78 (2009), no. 3, 275-295, DOI 10.1002/nme.2485. MR2542506 (2010m:65223)

[56] P.-A. Raviart and J. M. Thomas, A mixed finite element method for 2nd order elliptic problems, Naz. delle Ricerche (C.N.R.), Rome, 1975), Springer, Berlin, 1977, pp. 292-315. Lecture Notes in Math., Vol. 606. MR0483555 (58 \#3547)

[57] S.-C. Soon, Hybridizable discontinuous Galerkin methods for solid mechanics, Ph.D. thesis, University of Minnesota, 2008.

[58] S.-C. Soon, B. Cockburn, and Henryk K. Stolarski, A hybridizable discontinuous Galerkin method for linear elasticity, Internat. J. Numer. Methods Engrg. 80 (2009), no. 8, 1058-1092, DOI 10.1002/nme.2646. MR2589528 (2011a:74015)

[59] Rolf Stenberg, A family of mixed finite elements for the elasticity problem, Numer. Math. 53 (1988), no. 5, 513-538, DOI 10.1007/BF01397550. MR.954768 (89h:65192)

[60] Rolf Stenberg, Postprocessing schemes for some mixed finite elements, RAIRO Modél. Math. Anal. Numér. 25 (1991), no. 1, 151-167 (English, with French summary). MR.1086845 (92a:65303)

[61] Jacek Szarski, Differential inequalities, Monografie Matematyczne, Tom 43, Państwowe Wydawnictwo Naukowe, Warsaw, 1965. MR0190409 (32 \#7822)

[62] Liu Y. and Wei X., Finite-difference numerical modeling with even-order accuracy in twophase anisotropic media, Applied Geophysics 5 (2008), 107-114.

[63] Abraham Zemui, Fourth order symmetric finite difference schemes for the acoustic wave equation, BIT 45 (2005), no. 3, 627-651, DOI 10.1007/s10543-005-0021-4. MR2189769 (2006h:65131)

School of Mathematics, University of Minnesota, 206 Church Street S.E., MinneapoLIS, Minnesota 55455

E-mail address: cockburn@math.umn.edu

School of Mathematics, University of Minnesota, 206 Church Street S.E., MinneapoLis, Minnesota 55455

E-mail address: vqb@math.umn.edu 